\title{
On local motion of a general compressible viscous heat conducting fluid bounded by a free surface
}

\author{
by EWA ZADRZYŃSKA (Łódź) \\ and Wojciech M. ZAJA̧CZKowski (Warszawa)
}

\begin{abstract}
The motion of a viscous compressible heat conducting fluid in a domain in $\mathbb{R}^{3}$ bounded by a free surface is considered. We prove local existence and uniqueness of solutions in Sobolev-Slobodetskiĭ spaces in two cases: with surface tension and without it.
\end{abstract}

1. Introduction. In this paper we consider the motion of a viscous heat conducting fluid in a bounded domain $\Omega_{t} \subset \mathbb{R}^{3}$ with a free boundary $S_{t}$. Let $v=v(x, t)$ be the velocity of the fluid (i.e. $\left.v=\left(v_{1}, v_{2}, v_{3}\right)\right), \varrho=\varrho(x, t)$ the density, $\vartheta=\vartheta(x, t)$ the temperature, $f=f(x, t)$ the external force field per unit mass, $r=r(x, t)$ the efficiency of heat sources per unit mass, $p=p(\varrho, \vartheta)$ the pressure, $\mu$ and $\nu$ the constant viscosity coefficients, $\sigma$ the constant coefficient of surface tension, $\kappa$ the constant coefficient of heat conductivity, $c_{v}=c_{v}(\varrho, \vartheta)$ the specific heat at constant volume, $p_{0}$ the external (constant) pressure. Then the problem is described by the following system (see [4], Chs. 2 and 5):

$$
\begin{array}{ll}
\varrho\left(v_{t}+v \cdot \nabla v\right)+\nabla p-\mu \Delta v-\nu \nabla \operatorname{div} v=\varrho f & \text { in } \widetilde{\Omega}^{T}, \\
\varrho_{t}+\operatorname{div}(\varrho v)=0 & \text { in } \widetilde{\Omega}^{T}, \\
\varrho c_{v}\left(\vartheta_{t}+v \cdot \nabla \vartheta\right)+\vartheta p_{\vartheta} \operatorname{div} v-\kappa \Delta \vartheta & \\
\quad-\frac{\mu}{2} \sum_{i, j=1}^{3}\left(v_{i, x_{j}}+v_{j, x_{i}}\right)^{2}-(\nu-\mu)(\operatorname{div} v)^{2}=\varrho r & \text { in } \widetilde{\Omega}^{T}, \\
\mathbb{T} \cdot \bar{n}-\sigma H \bar{n}=-p_{0} \bar{n} & \text { on } \widetilde{S}^{T},
\end{array}
$$

1991 Mathematics Subject Classification: 35A05, 35R35, 76N10.

Key words and phrases: free boundary, compressible viscuous heat conducting fluid, local existence, anisotropic Sobolev spaces.

Research supported by KBN grant no. 211419101. 


$$
\begin{array}{ll}
v \cdot \bar{n}=-\frac{\phi_{t}}{|\nabla \phi|} & \text { on } \widetilde{S}^{T}, \\
\frac{\partial \vartheta}{\partial n}=\widetilde{\vartheta} & \text { on } \widetilde{S}^{T}, \\
\left.v\right|_{t=0}=v_{0} & \text { in } \Omega, \\
\left.\varrho\right|_{t=0}=\varrho_{0} & \text { in } \Omega, \\
\left.\vartheta\right|_{t=0}=\vartheta_{0} & \text { in } \Omega,
\end{array}
$$

where $\phi(x, t)=0$ describes $S_{t}, \bar{n}$ is the unit outward vector normal to the boundary (i.e. $\bar{n}=\nabla \phi /|\nabla \phi|), \widetilde{\Omega}^{T}=\bigcup_{t \in(0, T)} \Omega_{t} \times\{t\}, \Omega_{t}$ is the domain of the drop at time $t$ and $\Omega_{0}=\Omega$ is its initial domain, $\widetilde{S}^{T}=\bigcup_{t \in(0, T)} S_{t} \times\{t\}$. Finally, $\mathbb{T}=\mathbb{T}(v, p)$ denotes the stress tensor of the form

$$
\begin{aligned}
\mathbb{T} & =\left\{T_{i j}\right\}=\left\{-p \delta_{i j}+\mu\left(v_{i, x_{j}}+v_{j, x_{i}}\right)+(\nu-\mu) \delta_{i j} \operatorname{div} v\right\} \\
& \equiv\left\{-p \delta_{i j}+D_{i j}(v)\right\},
\end{aligned}
$$

where $i, j=1,2,3, \mathbb{D}=\mathbb{D}(v)=\left\{D_{i j}\right\}$ is the deformation tensor. Moreover, thermodynamic considerations imply that $c_{v}>0, \kappa>0, \nu \geq \frac{1}{3} \mu>0$. By $H$ we denote the double mean curvature of $S_{t}$ which is negative for convex domains and can be expressed in the form

$$
H \bar{n}=\Delta_{S_{t}}(t) x, \quad x=\left(x_{1}, x_{2}, x_{3}\right),
$$

where $\Delta_{S_{t}}(t)$ is the Laplace-Beltrami operator on $S_{t}$. Let $S_{t}$ be given by $x=x\left(s_{1}, s_{2}, t\right),\left(s_{1}, s_{2}\right) \in U \subset \mathbb{R}^{2}$, where $U$ is an open set. Then

$$
\begin{aligned}
\Delta_{S_{t}}(t) & =g^{-1 / 2} \frac{\partial}{\partial s_{\alpha}} g^{-1 / 2} \widehat{g}_{\alpha \beta} \frac{\partial}{\partial s_{\beta}} \\
& =g^{-1 / 2} \frac{\partial}{\partial s_{\alpha}} g^{1 / 2} g^{\alpha \beta} \frac{\partial}{\partial s_{\beta}} \quad(\alpha, \beta=1,2),
\end{aligned}
$$

where the summation convention over repeated indices is assumed, $g=$ $\operatorname{det}\left\{g_{\alpha \beta}\right\}_{\alpha, \beta=1,2}, g_{\alpha \beta}=x_{\alpha} \cdot x_{\beta}\left(x_{\alpha}=\partial x / \partial s_{\alpha}\right),\left\{g^{\alpha \beta}\right\}$ is the inverse matrix to $\left\{g_{\alpha \beta}\right\}$ and $\left\{\widehat{g}_{\alpha \beta}\right\}$ is the matrix of algebraic complements of $\left\{g_{\alpha \beta}\right\}$.

Let the domain $\Omega$ be given. Then by $(1.1)_{5}, \Omega_{t}=\left\{x \in \mathbb{R}^{3}: x=x(\xi, t)\right.$, $\xi \in \Omega\}$, where $x=x(\xi, t)$ is the solution of the Cauchy problem

$$
\frac{\partial x}{\partial t}=v(x, t),\left.\quad x\right|_{t=0}=\xi \in \Omega, \xi=\left(\xi_{1}, \xi_{2}, \xi_{3}\right) .
$$

Therefore the transformation $x=x(\xi, t)$ connects Eulerian $x$ and Lagrangian $\xi$ coordinates of the same fluid particle. Hence

$$
x=\xi+\int_{0}^{t} u(\xi, s) d s \equiv X_{u}(\xi, t),
$$


where $u(\xi, t)=v\left(X_{u}(\xi, t), t\right)$. Moreover, the kinematic boundary condition $(1.1)_{5}$ implies that the boundary $S_{t}$ is a material surface. Thus, if $\xi \in S=S_{0}$ then $X_{u}(\xi, t) \in S_{t}$ and $S_{t}=\left\{x: x=X_{u}(\xi, t), \xi \in S\right\}$.

By the equation of continuity $(1.1)_{2}$ and $(1.1)_{5}$ the total mass $M$ of the drop is conserved and the following relation between $\varrho$ and $\Omega_{t}$ holds:

$$
\int_{\Omega_{t}} \varrho(x, t) d x=M .
$$

The aim of this paper is to prove the local-in-time existence and uniqueness of solutions to problem (1.1) in Sobolev-Slobodetskil spaces (see definition in Sect. 2). In the case of compressible barotropic fluid the corresponding drop problem has been considered by W. M. Zajączkowski in [13] and [16], while papers [14] and [15] refer to the global existence of solution to the same drop problem. Local existence of solutions in the compressible barotropic case was also considered in [5], [6], [12], while in the incompressible barotropic case local existence is proved in [2] and [10].

This paper consists of four sections. In Section 2 notation and auxiliary results are presented. In Section 3 we prove the local existence and uniqueness of solution to problem (1.1) in the case $\sigma=0$. In this case there is no surface tension. Finally, Section 4 concerns the local existence and uniqueness of solution to problem (1.1) in the case $\sigma \neq 0$, i.e. when the shape of the free boundary $S_{t}$ of $\Omega_{t}$ is governed by surface tension.

2. Notations and auxiliary results. In Sections 3 and 4 of this paper we use the anisotropic Sobolev-Slobodetskiu spaces $W_{2}^{l, l / 2}\left(Q_{T}\right), l \in \mathbb{R}_{+}^{1}$ (see [3]), of functions defined in $Q_{T}$ where $Q_{T}=\Omega^{T} \equiv \Omega \times(0, T)\left(\Omega \subset \mathbb{R}^{3}\right.$ is a domain, $T \leq \infty)$ or $Q_{T}=S^{T} \equiv S \times(0, T), S=\partial \Omega$.

We define $W_{2}^{l, l / 2}\left(\Omega^{T}\right)$ as the space of functions $u$ such that

$$
\begin{aligned}
& \|u\|_{W_{2}^{l, l / 2}\left(\Omega^{T}\right)}=\left[\sum_{|\alpha|+2 i \leq[l]}\left\|D_{\xi}^{\alpha} \partial_{t}^{i} u\right\|_{L_{2}\left(\Omega^{T}\right)}^{2}\right. \\
& \quad+\sum_{|\alpha|+2 i=[l]}\left(\int_{0}^{T} \int_{\Omega} \int_{\Omega} \frac{\left|D_{\xi}^{\alpha} \partial_{t}^{i} u(\xi, t)-D_{\xi^{\prime}}^{\alpha} \partial_{t}^{i} u\left(\xi^{\prime}, t\right)\right|^{2}}{\left|\xi-\xi^{\prime}\right|^{3+2(l-[l])} d \xi d \xi^{\prime} d t}\right. \\
& \quad+\int_{\Omega} \int_{0}^{T} \int_{0}^{T} \frac{\left|D_{\xi}^{\alpha} \partial_{t}^{i} u(\xi, t)-D_{\xi}^{\alpha} \partial_{t^{\prime}}^{i} u\left(\xi, t^{\prime}\right)\right|^{2}}{\left.\left.\left|t-t^{\prime}\right|^{1+2(l / 2-[l / 2])} d t d t^{\prime} d \xi\right)\right]^{1 / 2}} \\
& \equiv\left[\sum_{|\alpha|+2 i \leq[l]}\left|D_{\xi}^{\alpha} \partial_{t}^{i} u\right|_{2, \Omega^{T}}^{2}+\sum_{|\alpha|+2 i=[l]}\left(\left[D_{\xi}^{\alpha} \partial_{t}^{i} u\right]_{l-[l], 2, \Omega^{T}, \xi}^{2}\right.\right. \\
& \left.\left.\quad+\left[D_{\xi}^{\alpha} \partial_{t}^{i} u\right]_{l / 2-[l / 2], 2, \Omega^{T}, t}^{2}\right)\right]^{1 / 2}<\infty,
\end{aligned}
$$


where we use generalized (Sobolev) derivatives, $D_{\xi}^{\alpha}=\partial_{\xi_{1}}^{\alpha_{1}} \partial_{\xi_{2}}^{\alpha_{2}} \partial_{\xi_{3}}^{\alpha_{3}}, \partial_{\xi_{j}}^{\alpha_{j}}=$ $\partial^{\alpha_{j}} / \partial \xi_{j}^{\alpha_{j}}(j=1,2,3), \alpha=\left(\alpha_{1}, \alpha_{2}, \alpha_{3}\right)$ is a multiindex, $|\alpha|=\alpha_{1}+\alpha_{2}+\alpha_{3}$, $\partial_{t}^{i}=\partial^{i} / \partial t^{i}$ and $[l]$ is the integer part of $l$. In the case when $l$ is an integer the second terms in the above formulae must be omitted, while in the case of $l / 2$ being integer the last terms in the above formulae must be omitted as well.

Similarly to $W_{2}^{l, l / 2}\left(\Omega^{T}\right)$, using local mappings and a partition of unity we introduce the normed space $W_{2}^{l, l / 2}\left(S^{T}\right)$ of functions defined on $S^{T}=$ $S \times(0, T)$, where $S=\partial \Omega$.

We also use the usual Sobolev spaces $W_{2}^{l}(Q)$, where $l \in \mathbb{R}_{+}, Q=\Omega$ ( $\Omega \subset \mathbb{R}^{3}$ is a bounded domain) or $Q=S$. In the case $Q=\Omega$ the norm in $W_{2}^{l}(\Omega)$ is defined as follows:

$$
\begin{aligned}
\|u\|_{W_{2}^{l}(\Omega)}= & \left(\sum_{|\alpha| \leq[l]}\left\|D_{\xi}^{\alpha} u\right\|_{L_{2}(\Omega)}^{2}\right. \\
& \left.+\sum_{|\alpha|=[l]} \int_{\Omega} \int_{\Omega} \frac{\left|D_{\xi}^{\alpha} u(\xi)-D_{\xi^{\prime}}^{\alpha} u\left(\xi^{\prime}\right)\right|^{2}}{\left|\xi-\xi^{\prime}\right|^{3+2(l-[l])}} d \xi d \xi^{\prime}\right)^{1 / 2} \\
\equiv & \left(\sum_{|\alpha| \leq[l]}\left|D_{\xi}^{\alpha} u\right|_{2, \Omega}^{2}+\left[D_{\xi}^{\alpha} u\right]_{l-[l], 2, \Omega}^{2}\right)^{1 / 2}
\end{aligned}
$$

where the last term is omitted when $l$ is an integer. Similarly, by using local mappings and a partition of unity we define $W_{2}^{l}(S)$.

To simplify notation we write

$$
\begin{array}{ll}
\|u\|_{l, Q}=\|u\|_{W_{2}^{l, l / 2}(Q)} & \text { if } Q=\Omega^{T} \text { or } Q=S^{T}, l \geq 0 ; \\
\|u\|_{l, Q}=\|u\|_{W_{2}^{l}(Q)} & \text { if } Q=\Omega \text { or } Q=S, l \geq 0,
\end{array}
$$

and $W_{2}^{0,0}(Q)=W_{2}^{0}(Q)=L_{2}(Q)$. Moreover,

$$
\|u\|_{L_{p}(Q)}=|u|_{p, Q}, \quad 1 \leq p \leq \infty .
$$

Next introduce the space $\Gamma_{0}^{l, l / 2}(\Omega)$ with the norm

$$
\|u\|_{\Gamma_{0}^{l, l / 2}(\Omega)}=\sum_{|\alpha|+2 i \leq l}\left\|D_{\xi}^{\alpha} \partial_{t}^{i} u\right\|_{0, \Omega} \equiv|u|_{l, 0, \Omega}
$$

and the space $L_{p}\left(0, T ; \Gamma_{0}^{l, l / 2}(\Omega)\right)$ with the norm

$$
\|u\|_{L_{p}\left(0, T ; \Gamma_{0}^{l, l / 2}(\Omega)\right)} \equiv|u|_{l, 0, p, \Omega^{T}}, \quad \text { where } 1 \leq p \leq \infty .
$$

Moreover, let $C^{\alpha}\left(\Omega^{T}\right)(\alpha \in(0,1))$ denote the Hölder space with the norm

$$
\|u\|_{C^{\alpha}\left(\Omega^{T}\right)}=\sup _{\Omega^{T}} \frac{\left|u(\xi, t)-u\left(\xi^{\prime}, t^{\prime}\right)\right|}{\left(\sqrt{\left|\xi-\xi^{\prime}\right|^{2}+\left|t-t^{\prime}\right|^{2}}\right)^{\alpha}} ;
$$


let $C_{B}^{0}\left(\Omega^{T}\right)$ be the space of continuous bounded functions on $\Omega^{T}$ with the norm

$$
\|u\|_{C_{B}^{0}\left(\Omega^{T}\right)}=\sup _{\Omega^{T}}|u(\xi, t)|
$$

and let $C^{2,1}(Q)\left(Q \subset \mathbb{R}^{3} \times(0, T)\right)$ denote the space of functions $u$ such that $D_{\xi}^{\alpha} \partial_{t}^{i} u \in C^{0}(Q)$ for $|\alpha|+2 i \leq 2$.

Finally, the following seminorms are used:

$$
\mathbf{|} u \mathbf{|}_{\kappa, Q^{T}}=\left(\int_{0}^{T} \frac{|u|_{2, Q}^{2}}{t^{2 \kappa}} d t\right)^{1 / 2},
$$

where $Q=\Omega\left(\Omega \subset \mathbb{R}^{3}\right.$ is a bounded domain) or $Q=S$, and $\kappa \in(0,1)$;

$$
[u]_{l, 2, Q}=[u]_{l, 2, Q, \xi}+[u]_{l, 2, Q, t},
$$

where

$$
\begin{aligned}
{[u]_{l, 2, Q, \xi} } & =\sum_{|\alpha|+2 i=[l]}\left[D_{\xi}^{\alpha} \partial_{t}^{i} u\right]_{l-[l], 2, Q, \xi}, \\
{[u]_{l, 2, Q, t} } & =\sum_{|\alpha|+2 i=[l]}\left[D_{\xi}^{\alpha} \partial_{t}^{i} u\right]_{l / 2-[l / 2], 2, Q, t},
\end{aligned}
$$

$Q=\Omega \times J\left(\Omega \subset \mathbb{R}^{3}\right.$ is a domain, $J=(0, T)$ or $\left.J=(-\infty, T)\right)$ or $Q=S \times J$.

In the case when $J=(0, T)$ the seminorms $\left[D_{\xi}^{\alpha} \partial_{t}^{i} u\right]_{l-[l], 2, Q, \xi}$ and $\left[D_{\xi}^{\alpha} \partial_{t}^{i} u\right]_{l / 2-[l / 2], 2, Q, t}$ are defined in (2.1). In the case when $J=(-\infty, T)$ we define the above seminorms in the same way.

Let $X$ be whichever of the function spaces mentioned above. We say that a vector-valued function $u=\left(u_{1}, \ldots, u_{\nu}\right)$ belongs to $X$ if $u_{i} \in X$ for any $1 \leq i \leq \nu$.

In the sequel we shall use various notations for derivatives of $u$ (where $u$ is a scalar- or vector-valued function $\left.u=\left(u_{1}, u_{2}, u_{3}\right)\right)$. If $u$ is a scalar-valued function we denote by $D_{\xi}^{k} u$ (where $\xi \in \Omega \subset \mathbb{R}^{3}$ ) the vector of all derivatives of $u$ of order $k$, i.e. $D_{\xi}^{k} u=\left(D_{\xi}^{\alpha} u\right)_{|\alpha|=k}$. Similarly, if $u=\left(u_{1}, u_{2}, u_{3}\right)$ we denote by $D_{\xi}^{k} u$ the vector $\left(D_{\xi}^{\alpha} u_{j}\right)_{|\alpha|=k, j=1,2,3}$. By $D_{\xi, t}^{k} u$ we denote the vector $\left(D_{\xi}^{\alpha} \partial_{t}^{i} u_{j}\right)_{|\alpha|+2 i=k, j=1,2,3}$ in the case when $u=\left(u_{1}, u_{2}, u_{3}\right)$ and the vector $\left(D_{\xi}^{\alpha} \partial_{t}^{i} u\right)_{|\alpha|+2 i=k}$ in the scalar case. Hence

$$
\left|D_{\xi}^{k} u\right|=\sum_{|\alpha|=k}\left|D_{\xi}^{\alpha} u\right| \quad \text { and } \quad\left|D_{\xi, t}^{k} u\right|=\sum_{|\alpha|+2 i=k}\left|D_{\xi}^{\alpha} \partial_{t}^{i} u\right| .
$$

We also use the notation $\nabla_{\xi} u \equiv D_{\xi}^{1} u$ or $u_{\xi} \equiv D_{\xi}^{1} u$.

Next, we denote by $u \cdot v$ either the scalar product of vectors $u$ and $v$, or the product of matrices $u$ and $v$. 
Finally, we denote by $D_{\xi, t}^{k} u D_{\xi, t}^{l} v$ the following number:

$$
D_{\xi, t}^{k} u D_{\xi, t}^{l} v=\sum_{\substack{|\alpha|+2 i=k \\|\beta|+2 j=l}} \sum_{m=1}^{p} \sum_{n=1}^{s} D_{\xi}^{\alpha} \partial_{t}^{i} u_{m} D_{\xi}^{\beta} \partial_{t}^{j} v_{n}
$$

where $u=\left(u_{1}, \ldots, u_{p}\right), v=\left(v_{1}, \ldots, v_{s}\right)(k \geq 0, l \geq 0, p>1, s>1)$. The product of more than two such factors is defined similarly.

We use the following lemmas.

LEMMA 2.1. The following imbedding holds:

$$
W_{r}^{l}(\Omega) \subset L_{p}^{\alpha}(\Omega) \quad\left(\Omega \subset \mathbb{R}^{3}\right),
$$

where $|\alpha|+3 / r-3 \leq l, l \in \mathbb{Z}, 1 \leq p, r \leq \infty ; L_{p}^{\alpha}(\Omega)$ is the space of functions $u$ such that $\left|D_{\xi}^{\alpha} u\right|_{p, \Omega}<\infty ; W_{r}^{l}(\Omega)$ is the Sobolev space.

Moreover, the following interpolation inequalities hold:

$$
\left|D_{\xi}^{\alpha} u\right|_{p, \Omega} \leq c \varepsilon^{1-\kappa}\left|D_{\xi}^{l} u\right|_{r, \Omega}+c \varepsilon^{-\kappa}|u|_{r, \Omega},
$$

where $\kappa=|\alpha| / l+3 /(l r)-3 /(l p)<1, \varepsilon$ is a parameter and $c>0$ is a constant independent of $u$ and $\varepsilon$;

$$
\left|D_{\xi}^{\alpha} u\right|_{q, S} \leq c \varepsilon^{1-\kappa}\left|D_{\xi}^{l} u\right|_{r, \Omega}+c \varepsilon^{-\kappa}|u|_{r, \Omega},
$$

where $\kappa=|\alpha| / l+3 /(l r)-2 /(l q)<1$, $\varepsilon$ is a parameter and $c>0$ is a constant independent of $u$ and $\varepsilon$.

Lemma 2.1 follows from Theorem 10.2 of [3].

LEMMA 2.2 (see [7]). For sufficiently regular $u$ we have

$$
\left\|\partial_{t}^{i} u(t)\right\|_{2 l-1-2 i, \Omega} \leq c\left(\|u\|_{2 l, \Omega^{T}}+\left\|\partial_{t}^{i} u(0)\right\|_{2 l-1-2 i, \Omega}\right),
$$

where $0 \leq 2 i \leq 2 l-1, l \in \mathbb{N}$ and $c>0$ is a constant independent of $T$.

LEMMA 2.3. Let $u(\xi, t)=0$ for $t \leq 0$. Then

$$
\begin{aligned}
\int_{-\infty}^{T} d t \int_{-\infty}^{T} d t^{\prime} \frac{\left|u(\xi, t)-u\left(\xi, t^{\prime}\right)\right|_{2, Q}^{2}}{\left|t-t^{\prime}\right|^{1+2 \alpha}} \leq & \int_{0}^{T} d t \int_{0}^{T} d t^{\prime} \frac{\left|u(\xi, t)-u\left(\xi, t^{\prime}\right)\right|_{2, Q}^{2}}{\left|t-t^{\prime}\right|^{1+2 \alpha}} \\
& +\frac{1}{\alpha} \int_{0}^{T} \frac{|u(t)|_{2, Q}^{2}}{t^{2 \alpha}} d t,
\end{aligned}
$$

where $Q=\Omega\left(\Omega \subset \mathbb{R}^{3}\right.$ is a domain $)$ or $Q=S=\partial \Omega$, and $\alpha \in \mathbb{R}$.

LEMmA 2.4. Let $\tau \in(0,1)$. Then for all $u \in W_{2}^{0, \tau / 2}\left(\Omega^{T}\right)$,

(2.6) $\int_{0}^{T}|u|_{2, \Omega}^{2} \frac{d t}{t^{\tau}} \leq c_{1} \int_{0}^{T} d t \int_{0}^{T} d t^{\prime} \frac{\left|u(\cdot, t)-u\left(\cdot, t^{\prime}\right)\right|_{2, \Omega}^{2}}{\left|t-t^{\prime}\right|^{1+\tau}}+c_{2} T^{-\tau} \int_{0}^{T}|u|_{2, \Omega}^{2} d t$, 
where $c_{1}, c_{2}$ do not depend on $T$ and $u$. For $T=\infty$ the last term in (2.6) vanishes.

This was shown in [11], Lemma 6.3.

3. Local existence in the case $\sigma=0$. In order to prove local existence of solutions of (1.1) we rewrite it in the Lagrangian coordinates introduced by (1.5) and (1.6):

$$
\begin{array}{ll}
\eta u_{t}-\mu \nabla_{u}^{2} u-\nu \nabla_{u} \nabla_{u} \cdot u+\nabla_{u} p(\eta, \gamma)=\eta g & \text { in } \Omega^{T}, \\
\eta_{t}+\eta \nabla_{u} \cdot u=0 & \text { in } \Omega^{T}, \\
\eta c_{v}(\eta, \gamma) \gamma_{t}-\kappa \nabla_{u}^{2} \gamma=-\gamma p_{\gamma}(\eta, \gamma) \nabla_{u} \cdot u & \\
\quad+\frac{\mu}{2} \sum_{i, j=1}^{3}\left(\xi_{x_{i}} \cdot \nabla_{\xi} u_{j}+\xi_{x_{j}} \cdot \nabla_{\xi} u_{i}\right)^{2} & \\
\quad+(\nu-\mu)\left(\nabla_{u} \cdot u\right)^{2}+\eta k & \text { in } \Omega^{T}, \\
\mathbb{T}_{u}(u, p) \cdot \bar{n}=-p_{0} \bar{n} & \text { on } S^{T}, \\
\bar{n} \cdot \nabla_{u} \gamma=\widetilde{\gamma} & \text { on } S^{T}, \\
\left.u\right|_{t=0}=v_{0} & \text { in } \Omega, \\
\left.\eta\right|_{t=0}=\rho_{0} & \text { in } \Omega, \\
\left.\gamma\right|_{t=0}=\vartheta_{0} & \text { in } \Omega,
\end{array}
$$

where $u(\xi, t)=v\left(X_{u}(\xi, t), t\right), \gamma(\xi, t)=\vartheta\left(X_{u}(\xi, t), t\right), \eta(\xi, t)=\rho\left(X_{u}(\xi, t), t\right)$, $g(\xi, t)=f\left(X_{u}(\xi, t), t\right), k(\xi, t)=r\left(X_{u}(\xi, t), t\right), \nabla_{u}=\xi_{x} \nabla_{\xi} \equiv\left\{\xi_{i x} \partial_{\xi_{i}}\right\}$,

$$
\begin{aligned}
\mathbb{T}_{u}(u, p) & =-p \mathbf{1}+\mathbb{D}_{u}(u), \\
\mathbb{D}_{u}(u) & =\left\{\mu\left(\xi_{k x_{i}} \partial_{\xi_{k}} u_{j}+\xi_{k x_{j}} \partial_{\xi_{k}} u_{i}\right)+(\nu-\mu) \delta_{i j} \nabla_{u} \cdot u\right\}
\end{aligned}
$$

(here the summation convention over repeated indices is assumed and $\mathbf{1}$ is the unit matrix) and $\widetilde{\gamma}(\xi, t)=\widetilde{\vartheta}\left(X_{u}(\xi, t), t\right)$.

Let $A=\left\{a_{i j}\right\}$ be the Jacobi matrix of the transformation $x=X_{u}(\xi, t)$, where $a_{i j}=\delta_{i j}+\int_{0}^{t} \partial_{\xi_{j}} u_{i}(\xi, \tau) d \tau$. Assuming that $\left|\nabla_{\xi} u\right|_{\infty, \Omega^{T}} \leq M$ we obtain

$$
0<c_{1}(1-M t)^{3} \leq \operatorname{det}\left\{x_{\xi}\right\} \leq c_{2}(1+M t)^{3}, \quad t \leq T,
$$

where $c_{1}, c_{2}>0$ are constants and $T>0$ is sufficiently small. Moreover,

$$
\operatorname{det} A=\exp \left(\int_{0}^{t} \nabla_{u} \cdot u d \tau\right)=\rho_{0} / \eta .
$$


Let $S_{t}$ be determined (at least locally) by the equation $\phi(x, t)=0$. Then $S$ is described by $\left.\phi(x(\xi, t), t)\right|_{t=0} \equiv \widetilde{\phi}(\xi)=0$. Thus, we have

$$
\bar{n}(x(\xi, t), t)=-\left.\frac{\nabla_{x} \phi(x, t)}{\left|\nabla_{x} \phi(x, t)\right|}\right|_{x=x(\xi, t)} \quad \text { and } \quad \bar{n}_{0}(\xi)=-\frac{\nabla_{\xi} \widetilde{\phi}(\xi)}{\left|\nabla_{\xi} \widetilde{\phi}(\xi)\right|} .
$$

First we consider the linear problems

$$
\begin{array}{ll}
L u \equiv u_{t}-\mu \nabla_{\xi}^{2} u-\nu \nabla_{\xi} \nabla_{\xi} \cdot u=F & \text { in } \Omega^{T}, \\
\mathbb{D}_{\xi}(u) \cdot \bar{n}_{0}=G & \text { on } S^{T}, \\
\left.u\right|_{t=0}=u_{0} & \text { in } \Omega
\end{array}
$$

$\left(\right.$ where $\left.\mathbb{D}_{\xi}(u)=\left\{\mu\left(\partial_{\xi_{i}} u_{j}+\partial_{\xi_{j}} u_{i}\right)+(\nu-\mu) \delta_{i j} \partial_{\xi_{k}} u_{k}\right\}\right)$ and

$$
\begin{array}{ll}
\gamma_{t}-\kappa \nabla_{\xi}^{2} \gamma=K & \text { in } \Omega^{T}, \\
\bar{n}_{0} \cdot \nabla_{\xi} \gamma=\widetilde{\gamma} & \text { on } S^{T}, \\
\left.\gamma\right|_{t=0}=\gamma_{0} & \text { in } \Omega .
\end{array}
$$

We assume

$$
\begin{gathered}
F \in W_{2}^{2,1}\left(\Omega^{T}\right), \quad G \in W_{2}^{3-1 / 2,3 / 2-1 / 4}\left(S^{T}\right), \\
\mid D_{\xi, t}^{2} G \mathbf{|}_{1 / 4, S^{T}}<\infty, \quad u_{0} \in W_{2}^{3}(\Omega)
\end{gathered}
$$

and

$$
\begin{gathered}
K \in W_{2}^{2,1}\left(\Omega^{T}\right), \quad \widetilde{\gamma} \in W_{2}^{3-1 / 2,3 / 2-1 / 4}\left(S^{T}\right), \\
\mid D_{\xi, t}^{2} \widetilde{\gamma} \mathbf{l}_{1 / 4, S^{T}}<\infty, \quad \gamma_{0} \in W_{2}^{3}(\Omega) .
\end{gathered}
$$

Moreover, we assume the following compatibility conditions:

$$
D_{\xi}^{\alpha}\left(\mathbb{D}_{\xi}(u(0)) \cdot \bar{n}_{0}-G(0)\right)=0, \quad|\alpha| \leq 1, \text { on } S,
$$

and

$$
D_{\xi}^{\alpha}\left(\bar{n}_{0} \cdot \nabla_{\xi} \gamma(0)-\widetilde{\gamma}(0)\right)=0, \quad|\alpha| \leq 1, \text { on } S .
$$

First we consider problem (3.3). Define functions $\psi_{i}$ for $i=0,1$ by

$$
\psi_{i}=\left.\partial_{t}^{i} u\right|_{t=0} \quad \text { in } \Omega .
$$

Hence

$$
\psi_{0}=u_{0}, \quad \psi_{1}=\mu \Delta u_{0}+\nu \nabla \operatorname{div} u_{0}+F(0) .
$$

LEMma 3.1. Let $\Omega \subset \mathbb{R}^{3}$ be either a halfspace or a bounded domain with smooth boundary $\partial \Omega$ and let $T \leq \infty$. Assume that

$$
\psi_{0} \in W_{2}^{3}(\Omega), \quad \psi_{1} \in W_{2}^{1}(\Omega) .
$$

Then there exists $v \in W_{2}^{4,2}\left(\Omega^{T}\right)$ such that

$$
\left.\partial_{t}^{i} v\right|_{t=0}=\psi_{i} \quad \text { in } \Omega(i=0,1)
$$


and

$$
\|v\|_{4, \Omega^{T}} \leq c\left(\left\|\psi_{0}\right\|_{3, \Omega}+\left\|\psi_{1}\right\|_{1, \Omega}\right),
$$

where $c>0$ is a constant independent of $T$.

Pro of. Using the Hestenes-Whitney method (see [1]) we can extend $\psi_{0}$ and $\psi_{1}$ to functions $\widetilde{\psi}_{0} \in W_{2}^{3}\left(\mathbb{R}^{3}\right)$ and $\widetilde{\psi}_{1} \in W_{2}^{1}\left(\mathbb{R}^{3}\right)$ such that

$$
\left\|\widetilde{\psi}_{0}\right\|_{3, \mathbb{R}^{3}} \leq c\left\|\psi_{0}\right\|_{3, \Omega}, \quad\left\|\widetilde{\psi}_{1}\right\|_{1, \mathbb{R}^{3}} \leq c\left\|\psi_{1}\right\|_{1, \Omega},
$$

where $c=c(\Omega)$. Then from [16] (Lemma 6.5) we deduce that there exists $\widetilde{v} \in W_{2}^{4,2}\left(\mathbb{R}^{3} \times \mathbb{R}_{+}^{1}\right)$ such that

$$
\left.\partial_{t}^{i} \widetilde{v}\right|_{t=0}=\widetilde{\psi}_{i}, \quad i=0,1,
$$

and

(3.15) $\quad\|\widetilde{v}\|_{4, \mathbb{R}^{3} \times \mathbb{R}_{+}^{1}} \leq c\left(\left\|\widetilde{\psi}_{0}\right\|_{3, \mathbb{R}^{3}}+\left\|\widetilde{\psi}_{1}\right\|_{1, \mathbb{R}^{3}}\right) \leq c\left(\left\|\psi_{0}\right\|_{3, \Omega}+\left\|\psi_{1}\right\|_{1, \Omega}\right)$.

Therefore $v=\left.\widetilde{v}\right|_{\Omega^{T}}$ satisfies conditions (3.12) and (3.13).

Now we prove the following theorem.

THEOREM 3.1. Let $S \in W_{2}^{4-1 / 2}$ and let assumptions (3.5) and (3.7) be satisfied $(T<\infty)$. Then there exists a solution of (3.3) such that $u \in$ $W_{2}^{4,2}\left(\Omega^{T}\right)$ and

(3.16) $\quad\|u\|_{4, \Omega^{T}}$

$$
\leq c(T)\left(\|F\|_{2, \Omega^{T}}+\|G\|_{3-1 / 2, S^{T}}+\left.\left|D_{\xi, t}^{2} G \mathbf{|}_{1 / 4, S^{T}}+\right| u(0)\right|_{3,0, \Omega}\right),
$$

where $c$ is an increasing continuous function of $T$ and

$$
|u(0)|_{3,0, \Omega}=\left\|u_{0}\right\|_{3, \Omega}+\left\|\left.\partial_{t} u\right|_{t=0}\right\|_{1, \Omega} .
$$

P r o of. Introduce the function

$$
w=u-v,
$$

where $v$ is the function from Lemma 3.1. Then instead of (3.3) we obtain the problem

$$
\begin{array}{ll}
w_{t}-\mu \Delta w-\nu \nabla \operatorname{div} w=F-L v \equiv f & \text { in } \Omega^{T}, \\
\mathbb{D}_{\xi}(w) \cdot \bar{n}_{0}=G-\mathbb{D}_{\xi}(v) \cdot \bar{n}_{0} \equiv g & \text { on } S^{T}, \\
\left.w\right|_{t=0}=0 & \text { in } \Omega,
\end{array}
$$

where in view of the compatibility condition (3.7) we have

$$
f(0)=g(0)=0 .
$$

It is sufficient to consider problem (3.19) in $\Omega^{T} \equiv R^{T} \equiv \mathbb{R}_{+}^{3} \times[0, T]$ because using a partition of unity and appropriate norms (see [8], Sects. $20,21)$ we obtain the existence and the appropriate estimate of solutions of (3.19) in a bounded domain $\Omega$. 
Thus, consider problem (3.19) in $R^{T}$ and extend functions $f, g, w$ by zero for $t<0$ to functions $f_{1}, g_{1}, w_{1}$. Then instead of (3.19) we get the following boundary value problem:

$$
\begin{array}{ll}
L w_{1}=f_{1} & \text { in } \widetilde{D}_{4}(T)=\mathbb{R}_{+}^{3} \times(-\infty, T], \\
\mathbb{D}_{\xi}\left(w_{1}\right) \cdot \bar{n}_{0}=g_{1} & \text { in } \widetilde{E}_{3}(T)=\mathbb{R}^{2} \times(-\infty, T] .
\end{array}
$$

Next using the Hestenes-Whitney method extend $f_{1}$ and $g_{1}$ to functions $f_{2}$ and $g_{2}$ defined on $\mathbb{R}_{+}^{3} \times(-\infty, \infty)$ and $\mathbb{R}^{2} \times(-\infty, \infty)$, respectively. Then instead of (3.21) we have the problem

$$
\begin{array}{ll}
L w_{2}=f_{2} & \text { in } \widetilde{D}_{4}=\mathbb{R}_{+}^{3} \times(-\infty, \infty), \\
\mathbb{D}_{\xi}\left(w_{2}\right) \cdot \bar{n}_{0}=g_{2} & \text { in } \widetilde{E}_{3}=\mathbb{R}^{2} \times(-\infty, \infty),
\end{array}
$$

and the estimate

$$
\left\|f_{2}\right\|_{2, \widetilde{D}_{4}} \leq c\left\|f_{1}\right\|_{2, \widetilde{D}_{4}(T)} .
$$

Next we extend $f_{2}$ by the Hestenes-Whitney method to a function $f_{3} \in$ $W_{2}^{4,2}\left(\mathbb{R}^{4}\right)$ such that

$$
\left\|f_{3}\right\|_{2, \mathbb{R}^{4}} \leq c\left\|f_{2}\right\|_{2, \widetilde{D}_{4}}
$$

Consider now the system

$$
L w_{3}=f_{3} \quad \text { in } \mathbb{R}^{4} .
$$

By potential techniques (see [8], Sections 12 and 21) and (3.23), (3.24) there exists a solution $w_{3} \in W_{2}^{4,2}\left(\mathbb{R}^{4}\right)$ of $(3.25)$ and

$$
\left\|w_{3}\right\|_{4, \mathbb{R}^{4}} \leq c(T)\|f\|_{2, R^{T}},
$$

where $c(T)$ is an increasing function of $T$.

Introduce the function

$$
w_{4}=w_{2}-w_{3}
$$

By (3.22) and (3.25) we have

$$
\begin{array}{ll}
L w_{4}=0 & \text { in } \widetilde{D}_{4}, \\
\mathbb{D}_{\xi}\left(w_{4}\right) \cdot \bar{n}_{0}=g_{2}-\mathbb{D}_{\xi}\left(w_{3}\right) \cdot \bar{n}_{0} \equiv g_{3} & \text { in } \widetilde{E}_{3} .
\end{array}
$$

Again by potential techniques there exists a solution $w_{4} \in W_{2}^{4,2}\left(\widetilde{D}_{4}\right)$ of (3.28) and

$$
\begin{aligned}
\left\|w_{4}\right\|_{4, \widetilde{D}_{4}} & \leq c\left[g_{3}\right]_{3-1 / 2,2, \widetilde{E}_{3}} \leq c\left(\left[g_{2}\right]_{3-1 / 2,2, \widetilde{E}_{3}}+\left\|w_{3}\right\|_{4, \widetilde{D}_{4}}\right) \\
& \leq c(T)\left(\left[g_{1}\right]_{3-1 / 2,2}, \widetilde{E}_{3}(T)+\|f\|_{2, R^{T}}\right),
\end{aligned}
$$

where we used (3.26) and the Hestenes-Whitney method for $g_{1}$. Hence 


$$
\left\|w_{4}\right\|_{4, R^{T}} \leq c(T)\left(\left[g_{1}\right]_{3-1 / 2,2, \widetilde{E}_{3}(T)}+\|f\|_{2, R^{T}}\right) .
$$

Further, using Lemma 2.3 we have

$$
\begin{aligned}
{\left[g_{1}\right]_{3-1 / 2,2, \widetilde{E}_{3}(T)}=} & \sum_{|\alpha|=2}\left[D_{\xi}^{\alpha} g_{1}\right]_{1 / 2,2, \widetilde{E}_{3}(T), \xi}+\left[\partial_{t} g_{1}\right]_{1 / 2,2, \widetilde{E}_{3}(T), \xi} \\
& +\sum_{|\alpha|=2}\left[D_{\xi}^{\alpha} g_{1}\right]_{1 / 4,2, \widetilde{E}_{3}(T), t}+\left[\partial_{t} g_{1}\right]_{1 / 4,2, \widetilde{E}_{3}(T), t} \\
\leq & c\left\{\sum_{|\alpha|=2}\left[D_{\xi}^{\alpha} g\right]_{1 / 2,2, \mathbb{R}^{2} \times[0, T], \xi}+\left[\partial_{t} g\right]_{1 / 2,2, \mathbb{R}^{2} \times[0, T], \xi}\right. \\
& +\sum_{|\alpha|=2}\left[D_{\xi}^{\alpha} g\right]_{1 / 4,2, \mathbb{R}^{2} \times[0, T], t}+\left[\partial_{t} g\right]_{1 / 4,2, \mathbb{R}^{2} \times[0, T], t} \\
& \left.+\left(\int_{0}^{T} \frac{\left|D_{\xi, t}^{2} g\right|_{2, \mathbb{R}^{2}}^{2}}{t^{1 / 2}} d t\right)^{1 / 2}\right\} \\
\leq & c\left(\|g\|_{3-1 / 2, S^{T}}+\left|D_{\xi, t}^{2} g\right|_{1 / 4, S^{T}}\right),
\end{aligned}
$$

where $S^{T}=\mathbb{R}^{2} \times[0, T]$.

Taking into account the right-hand side of (3.19) and (3.18), (3.21), (3.22), (3.25)-(3.30) we conclude that there exists a solution $u \in W_{2}^{4,2}\left(\Omega^{T}\right)$ of (3.3) satisfying

$$
\begin{aligned}
& \|u\|_{4, R^{T}} \\
& \leq c(T)\left(\|F\|_{2, R^{T}}+\|v\|_{4, R^{T}}+\|G\|_{3-1 / 2, S^{T}}\right. \\
& \left.\quad+\left|D_{\xi, t}^{2} G \mathbf{|}_{1 / 4, S^{T}}+\left\|v_{\xi} \bar{n}_{0}\right\|_{3-1 / 2, S^{T}}+\right| \mathbf{I} D_{\xi, t}^{2}\left(v_{\xi} \bar{n}_{0}\right) \mathbf{I}_{1 / 4, S^{T}}\right),
\end{aligned}
$$

where

$$
v_{\xi} \bar{n}_{0}=\sum_{i, j, k=1}^{3} v_{i \xi_{j}} n_{0 k} .
$$

By Lemma 3.1 we have

$$
\|v\|_{4, R^{T}} \leq c\left(\left\|\phi_{0}\right\|_{3, \mathbb{R}_{+}^{3}}+\left\|\phi_{1}\right\|_{1, \mathbb{R}_{+}^{3}}\right)
$$

and

(3.33) $\quad\left\|v_{\xi} \bar{n}_{0}\right\|_{3-1 / 2, S^{T}} \leq\left\|\widetilde{v}_{\xi} \bar{n}_{0}\right\|_{3-1 / 2, S \times(0, \infty)} \leq c\left\|\widetilde{v}_{\xi}\right\|_{3, \mathbb{R}_{+}^{3} \times(0, \infty)}$

$$
\leq c\|v\|_{4, R^{T}} \leq c\left(\left\|\phi_{0}\right\|_{3, \mathbb{R}_{+}^{3}}+\left\|\phi_{1}\right\|_{1, \mathbb{R}_{+}^{3}}\right),
$$

where we have used the fact that $S \in W_{2}^{4-1 / 2}$.

It remains to estimate $\mid D_{\xi, t}^{2}\left(v_{\xi} \bar{n}_{0}\right) \mathbf{I}_{1 / 4, S^{T}}$. We have 


$$
\begin{aligned}
& \mathbf{|} D_{\xi, t}^{2}\left(v_{\xi} \bar{n}_{0}\right) \mathbf{I}_{1 / 4, S^{T}} \\
= & \left(\int_{0}^{T} \frac{\left|D_{\xi}^{2} \bar{n}_{0} D_{\xi}^{1} v+D_{\xi}^{1} \bar{n}_{0} D_{\xi}^{2} v+\bar{n}_{0} D_{\xi}^{3} v+\bar{n}_{0} \partial_{t} D_{\xi}^{1} v\right|_{2, S}^{2}}{t^{1 / 2}} d t\right)^{1 / 2} \\
\leq & c\left(\int_{0}^{T} \frac{\left|D_{\xi}^{2} \bar{n}_{0}\right|_{4, S}^{2}\left|D_{\xi}^{1} v\right|_{4, S}^{2}+\left|D_{\xi}^{2} v\right|_{2, S}^{2}+\left|D_{\xi}^{3} v\right|_{2, S}^{2}+\left|\partial_{t} D_{\xi}^{1} v\right|_{2, S}^{2}}{t^{1 / 2}} d t\right)^{1 / 2},
\end{aligned}
$$

where we have used the fact that $S \in W_{2}^{4-1 / 2}$ and Lemma 2.1, and where the products are understood in the sense of (2.2).

Next, by Lemma 2.1 we get

$$
\int_{0}^{T} \frac{\left|D_{\xi}^{2} \bar{n}_{0}\right|_{4, S}^{2}\left|D_{\xi}^{1} v\right|_{4, S}^{2}}{t^{1 / 2}} d t \leq c \int_{0}^{T} \frac{\left\|D_{\xi}^{1} v\right\|_{1, S}^{2}}{t^{1 / 2}} d t .
$$

Hence in view of Lemma 2.4, (3.34) yields

$\mathbf{|} D_{\xi, t}^{2}\left(v_{\xi} \bar{n}_{0}\right) \mathbf{|}_{1 / 4, S^{T}}$

$$
\begin{aligned}
\leq & c\left(\int_{0}^{\infty} \frac{\left|D_{\xi}^{1} \widetilde{v}\right|_{2, S}^{2}+\left|D_{\xi}^{2} \widetilde{v}\right|_{2, S}^{2}+\left|D_{\xi}^{3} \widetilde{v}\right|_{2, S}^{2}+\left|D_{\xi}^{1} \partial_{t} \widetilde{v}\right|_{2, S}^{2}}{t^{1 / 2}} d t\right)^{1 / 2} \\
\leq & c_{1}\left[\int _ { 0 } ^ { \infty } d t \int _ { 0 } ^ { \infty } d t ^ { \prime } \left(\frac{\left|D_{\xi}^{1} \widetilde{v}(\xi, t)-D_{\xi}^{1} \widetilde{v}\left(\xi, t^{\prime}\right)\right|_{2, S}^{2}}{\left|t-t^{\prime}\right|^{1+1 / 2}}\right.\right. \\
& +\frac{\left|D_{\xi}^{2} \widetilde{v}(\xi, t)-D_{\xi}^{2} \widetilde{v}\left(\xi, t^{\prime}\right)\right|_{2, S}^{2}}{\left|t-t^{\prime}\right|^{1+1 / 2}}+\frac{\left|\partial_{t} D_{\xi}^{1} \widetilde{v}(\xi, t)-\partial_{t^{\prime}} D_{\xi}^{1} \widetilde{v}\left(\xi, t^{\prime}\right)\right|_{2, S}^{2}}{\left|t-t^{\prime}\right|^{1+1 / 2}} \\
& \left.\left.+\frac{\left|D_{\xi}^{3} \widetilde{v}(\xi, t)-D_{\xi}^{3} \widetilde{v}\left(\xi, t^{\prime}\right)\right|_{2, S}^{2}}{\left|t-t^{\prime}\right|^{1+1 / 2}}\right)\right]^{1 / 2} .
\end{aligned}
$$

Therefore

$$
\mathbf{|} D_{\xi, t}^{2}\left(v_{\xi} \bar{n}_{0}\right) \mathbf{I}_{1 / 4, S^{T}} \leq c\|v\|_{4, R^{T}},
$$

where we have used Theorem 5.1 from [8].

Taking into account (3.31)-(3.33) and (3.35) we get (3.16). This completes the proof of the theorem.

In the same way we can prove

TheOREM 3.2. Let $S \in W_{2}^{4-1 / 2}$ and let assumptions (3.6) and (3.8) be satisfied $(T<\infty)$. Then there exists a solution of (3.4) such that $\gamma \in$ $W_{2}^{4,2}\left(\Omega^{T}\right)$ and

$$
\begin{aligned}
& \|\gamma\|_{4, \Omega^{T}} \\
& \leq c(T)\left(\|K\|_{2, \Omega^{T}}+\|\widetilde{\gamma}\|_{3-1 / 2, S^{T}}+\left|\mathbf{I} D_{\xi, t}^{2} \widetilde{\gamma}\right|_{1 / 4, S^{T}}+|\gamma(0)|_{3,0, \Omega}\right),
\end{aligned}
$$


where $c$ is an increasing function of $T$ and

$$
|\gamma(0)|_{3,0, \Omega}=\left\|\gamma_{0}\right\|_{3, \Omega}+\left\|\left.\partial_{t}^{1} \gamma\right|_{t=0}\right\|_{1, \Omega} .
$$

Now we have to consider the following problems:

$$
\begin{array}{ll}
\eta u_{t}-\mu \nabla_{\xi}^{2} u-\nu \nabla_{\xi} \nabla_{\xi} \cdot u=F & \text { in } \Omega^{T}, \\
\mathbb{D}_{\xi}(u) \cdot \bar{n}_{0}=G & \text { on } S^{T}, \\
\left.u\right|_{t=0}=u_{0} & \text { in } \Omega
\end{array}
$$

and

$$
\begin{array}{ll}
\eta c_{v}(\eta, \beta) \gamma_{t}-\kappa \nabla_{\xi}^{2} \gamma=K & \text { in } \Omega^{T}, \\
\bar{n}_{0} \cdot \nabla_{\xi} \gamma=\widetilde{\gamma} & \text { on } S^{T}, \\
\left.\gamma\right|_{t=0}=\gamma_{0} & \text { in } \Omega .
\end{array}
$$

First we consider (3.38). The following theorem is proved in [15].

TheOrem 3.3. Assume that

$S \in W_{2}^{4-1 / 2}, F \in W_{2}^{2,1}\left(\Omega^{T}\right), G \in W_{2}^{3-1 / 2,3 / 2-1 / 4}\left(S^{T}\right)$,

$\mid D_{\xi, t}^{2} G \mathbf{|}_{1 / 4, S^{T}}<\infty, u_{0} \in W_{2}^{3}(\Omega)$,

$\eta \in L_{\infty}\left(0, T ; \Gamma_{0}^{2,1}(\Omega)\right) \cap C^{\alpha}\left(\Omega^{T}\right)(\alpha \in(0,1)), 1 / \eta \in L_{\infty}\left(\Omega^{T}\right)$.

Moreover, let the compatibility condition (3.7) be satisfied. Then there exists a unique solution $u \in W_{2}^{4,2}\left(\Omega^{T}\right)$ to problem (3.38) satisfying the estimate

$$
\begin{aligned}
\|u\|_{4, \Omega^{T}} \leq & \phi_{1}\left(|1 / \eta|_{\infty, \Omega^{T}},|\eta|_{\infty, \Omega^{T}}, T\right)\left[\|F\|_{2, \Omega^{T}}+\|G\|_{3-1 / 2, S^{T}}\right. \\
& \left.+\left|D_{\xi, t}^{2} G\right|_{1 / 4, S^{T}}+\phi_{2}\left(|\eta|_{2,0, \infty, \Omega^{T}},\|\eta\|_{C^{\alpha}\left(\Omega^{T}\right)}\right)\|u\|_{2, \Omega^{T}}\right] \\
& +\phi_{3}\left(|1 / \eta|_{\infty, \Omega^{T}}, T\right)|u(0)|_{3,0, \Omega},
\end{aligned}
$$

where $\phi_{i}(i=1,2,3)$ are nonnegative increasing continuous functions of their arguments.

Now we consider problem (3.39). Assume that $\eta \in L_{\infty}\left(0, T ; \Gamma_{0}^{2,1}(\Omega)\right)$ and $\beta \in W_{2}^{4,2}\left(\Omega^{T}\right)$. Applying Theorems 10.2 and 10.4 of [3] we find that $\eta \in C_{B}^{0}\left(\Omega^{T}\right)$ and $\beta \in C_{B}^{0}\left(\Omega^{T}\right)$. Therefore, there exists a bounded domain $V \subset \mathbb{R}^{2}$ such that $(\eta(\xi, t), \beta(\xi, t)) \in V$ for any $(\xi, t) \in \Omega^{T}$.

Lemma 3.2. Assume that $\eta \in L_{\infty}\left(0, T ; \Gamma_{0}^{2,1}(\Omega)\right) \cap C^{\alpha}\left(\Omega^{T}\right)$ (where $\alpha \in$ $(0,1 / 2)), 1 / \eta \in L_{\infty}\left(\Omega^{T}\right), \eta>0, \beta \in W_{2}^{4,2}\left(\Omega^{T}\right), 1 / \beta \in L_{\infty}\left(\Omega^{T}\right), \beta>0$, $c_{v} \in C^{2}\left(\mathbb{R}_{+}^{2}\right), c_{v}>0$. Then $\eta c_{v}(\eta, \beta) \in L_{\infty}\left(0, T ; \Gamma_{0}^{2,1}(\Omega)\right), \eta c_{v} \in C^{\alpha}\left(\Omega^{T}\right)$, $1 /\left(\eta c_{v}(\eta, \beta)\right) \in L_{\infty}\left(\Omega^{T}\right)$ and

(3.41) $\quad\left|\eta c_{v}(\eta, \beta)\right|_{2,0, \infty, \Omega^{T}}$

$$
\leq \psi_{1}\left(\left\|c_{v}\right\|_{C^{2}(\bar{V})},\left\|1 / c_{v}\right\|_{C^{0}(\bar{V})},|\eta|_{2,0, \infty, \Omega^{T}},\|\beta\|_{4, \Omega^{T}},|\beta(0)|_{3,0, \Omega}\right),
$$

$$
\left|\eta c_{v}(\eta, \beta)\right|_{\infty, \Omega^{T}} \leq\left\|c_{v}\right\|_{C^{0}(\bar{V})}|\eta|_{\infty, \Omega^{T}},
$$




$$
\begin{aligned}
& \left|1 /\left(\eta c_{v}(\eta, \beta)\right)\right|_{\infty, \Omega^{T}} \leq\left\|1 / c_{v}\right\|_{C^{0}(\bar{V})}|1 / \eta|_{\infty, \Omega^{T}}, \\
& \quad\left\|\eta c_{v}(\eta, \beta)\right\|_{C^{\alpha}\left(\Omega^{T}\right)} \\
& \quad \leq \psi_{2}\left(\left\|c_{v}\right\|_{C^{2}(\bar{V})},\|\eta\|_{C^{\alpha}\left(\Omega^{T}\right)},\|\beta\|_{4, \Omega^{T}},|\beta(0)|_{3,0, \Omega}, T\right),
\end{aligned}
$$

where $\psi_{i}(i=1,2)$ are nonnegative increasing continuous functions of their arguments.

Proof. By the assumptions we can choose $V$ such that $V \subset \mathbb{R}_{+}^{2}$. Thus (3.42) and (3.43) are obviously satisfied. In order to obtain (3.41) we calculate the derivatives $D_{\xi}^{\alpha} \partial_{t}^{i}\left(\eta c_{v}\right)$ (where $|\alpha|+2 i \leq 2$ ) and next we apply Lemmas 2.1 and 2.2. To prove (3.44) we also use Lemmas 2.1 and 2.2 and the Sobolev imbedding theorem.

Theorem 3.4. Assume that

$$
\begin{aligned}
& S \in W_{2}^{4-1 / 2}, K \in W_{2}^{2,1}\left(\Omega^{T}\right), \widetilde{\gamma} \in W_{2}^{3-1 / 2,3 / 2-1 / 4}\left(S^{T}\right), \gamma_{0} \in W_{2}^{3}(\Omega), \\
& \eta \in L_{\infty}\left(0, T ; \Gamma_{0}^{2,1}(\Omega)\right), 1 / \eta \in L_{\infty}\left(\Omega^{T}\right), \eta \in C^{\alpha}\left(\Omega^{T}\right)(0<\alpha<1 / 2), \\
& \eta>0, \beta \in W_{2}^{4,2}\left(\Omega^{T}\right), 1 / \beta \in L_{\infty}\left(\Omega^{T}\right), \beta>0, c_{v} \in C^{2}\left(\mathbb{R}_{+}^{2}\right), c_{v}>0 .
\end{aligned}
$$

Moreover, let the compatibility condition (3.8) be satisfied. Then there exists a unique solution $\gamma \in W_{2}^{4,2}\left(\Omega^{T}\right)$ to problem (3.39) satisfying the estimate

$$
\begin{aligned}
\|\gamma\|_{4, \Omega^{T}} \leq & \phi_{4}\left(\left\|c_{v}\right\|_{C^{0}(\bar{V})},\left\|1 / c_{v}\right\|_{C^{0}(\bar{V})},|\eta|_{\infty, \Omega^{T}},|1 / \eta|_{\infty, \Omega^{T}}, T\right) \\
& \times\left[\|K\|_{2, \Omega^{T}}+\|\widetilde{\gamma}\|_{3-1 / 2, S^{T}}+\left|D_{\xi, t}^{2} \widetilde{\gamma}\right|_{1 / 4, S^{T}}\right. \\
& +\phi_{5}\left(\left\|c_{v}\right\|_{C^{2}(\bar{V})},\left\|1 / c_{v}\right\|_{C^{0}(\bar{V})},|\eta|_{2,0, \infty, \Omega^{T}},\|\eta\|_{C^{\alpha}\left(\Omega^{T}\right)},\right. \\
& \left.\left.\|\beta\|_{4, \Omega^{T}},|\beta(0)|_{3,0, \Omega}, T\right)\|\gamma\|_{2, \Omega^{T}}\right] \\
& +\phi_{6}\left(\left\|1 / c_{v}\right\|_{C^{0}(\bar{V})},|1 / \eta|_{\infty, \Omega^{T}}, T\right)|\gamma(0)|_{3,0, \Omega},
\end{aligned}
$$

where $\phi_{i}(i=4,5,6)$ are nonnegative increasing continuous functions of their arguments.

Proof. Since by Lemma $3.2, \eta c_{v}(\eta, \beta) \in L_{\infty}\left(0, T ; \Gamma_{0}^{2,1}(\Omega)\right), \eta c_{v}(\eta, \beta) \in$ $C^{\alpha}\left(\Omega^{T}\right)(\alpha \in(0,1 / 2))$ and $1 /\left(\eta c_{v}(\eta, \beta)\right) \in L_{\infty}\left(\Omega^{T}\right)$, using the same argument as in Theorem 3.3 and inequalities (3.41)-(3.44) we prove the existence of a unique solution $\gamma \in W_{2}^{4,2}\left(\Omega^{T}\right)$ of problem (3.39) satisfying the estimate (3.45).

Now consider the problems

$$
\begin{array}{ll}
\eta u_{t}-\mu \nabla_{w}^{2} u-\nu \nabla_{w} \nabla_{w} \cdot u=F & \text { in } \Omega^{T}, \\
\mathbb{D}_{w}(u) \cdot \bar{n}=G & \text { on } S^{T}, \\
\left.u\right|_{t=0}=u_{0} & \text { in } \Omega,
\end{array}
$$


and

$$
\begin{array}{ll}
\eta c_{v}(\eta, \beta) \gamma_{t}-\kappa \nabla_{w}^{2} \gamma=K & \text { in } \Omega^{T}, \\
\bar{n} \cdot \nabla_{w} \gamma=\widetilde{\gamma} & \text { on } S^{T}, \\
\left.\gamma\right|_{t=0}=\gamma_{0} & \text { in } \Omega,
\end{array}
$$

where $\bar{n}=\bar{n}\left(X_{w}(\xi, t), t\right)$.

The following theorem concerns problem (3.46).

THEOREM 3.5. Assume that

$S \in W_{2}^{4-1 / 2}, F \in W_{2}^{2,1}\left(\Omega^{T}\right), G \in W_{2}^{3-1 / 2,3 / 2-1 / 4}\left(S^{T}\right)$,

$u_{0} \in W_{2}^{3}(\Omega), \eta \in L_{\infty}\left(0, T ; \Gamma_{0}^{2,1}(\Omega)\right), 1 / \eta \in L_{\infty}\left(\Omega^{T}\right)$,

$\eta \in C^{\alpha}\left(\Omega^{T}\right)(\alpha \in(0,1)), w \in W_{2}^{4,2}\left(\Omega^{T}\right) \cap C^{0}\left(0, T ; \Gamma_{0}^{3,3 / 2}(\Omega)\right), T<\infty$.

Moreover, let the compatibility condition (3.7) be satisfied. Assume that T is so small that

$$
\begin{aligned}
& T^{a}\left(\|w\|_{4, \Omega^{T}}+|w(0)|_{3,0, \Omega}\right) \\
\times & \phi_{7}\left(\int_{0}^{T}\left|w_{\xi}\right|_{\infty, \Omega} d t,|1 / \eta|_{\infty, \Omega^{T}},|\eta|_{\infty, \Omega^{T}},\|w\|_{4, \Omega^{T}},|w(0)|_{3,0, \Omega}, T\right) \leq \delta,
\end{aligned}
$$

where $\phi_{7}$ is an nonnegative nondecreasing continuous function of its arguments, $a>0$ is a constant and $\delta>0$ is sufficiently small. Then there exists a unique solution $u \in W_{2}^{4,2}\left(\Omega^{T}\right) \cap C^{0}\left(0, T ; \Gamma_{0}^{3,3 / 2}(\Omega)\right)$ to problem (3.46) satisfying the estimate

$$
\begin{aligned}
\|u\|_{4, \Omega^{T}} \leq & \phi_{1}\left(|1 / \eta|_{\infty, \Omega^{T}},|\eta|_{\infty, \Omega^{T}}, T\right)\left[\|F\|_{2, \Omega^{T}}+\|G\|_{3-1 / 2, S^{T}}\right. \\
& \left.+\left|D_{\xi, t}^{2} G\right|_{1 / 4, S^{T}}+\phi_{2}\left(|\eta|_{2,0, \infty, \Omega^{T}},\|\eta\|_{C^{\alpha}\left(\Omega^{T}\right)}\right)\|u\|_{2, \Omega^{T}}\right] \\
& +\phi_{3}^{\prime}\left(|1 / \eta|_{\infty, \Omega^{T}}, T\right)|u(0)|_{3,0, \Omega},
\end{aligned}
$$

where $\phi_{i}(i=1,2)$ are the functions from Theorem 3.3, and $\phi_{3}^{\prime}$ is a nonnegative increasing function.

Proof. In order to prove the existence of solutions to (3.46) we rewrite it as

$$
\begin{aligned}
& \eta u_{t}-\mu \nabla_{\xi}^{2} u-\nu \nabla_{\xi} \nabla_{\xi} \cdot u \\
& =F+\mu\left(\nabla_{w}^{2} u-\nabla_{\xi}^{2} u\right)+\nu\left(\nabla_{w} \nabla_{w} \cdot u-\nabla_{\xi} \nabla_{\xi} \cdot u\right) \\
& \equiv F+\widetilde{F} \quad \text { in } \Omega^{T}, \\
& \mathbb{D}_{\xi}(u) \cdot \bar{n}_{0}=G+\left(\mathbb{D}_{\xi}(u) \cdot \bar{n}_{0}-\mathbb{D}_{w}(u) \cdot \bar{n}\right) \\
& =\mathbb{D}_{\xi}(u) \cdot\left(\bar{n}_{0}-\bar{n}\right)+\left(\mathbb{D}_{\xi}(u)-\mathbb{D}_{w}(u)\right) \cdot \bar{n} \equiv G+\widetilde{G} \quad \text { on } S^{T}, \\
& \left.u\right|_{t=0}=u \\
& \text { in } \Omega \text {. }
\end{aligned}
$$


Using Theorem 3.3 we have the following estimate for solutions of (3.50):

$$
\begin{aligned}
\|u\|_{4, \Omega^{T}} \leq & \phi_{1}\left(|1 / \eta|_{\infty, \Omega^{T}},|\eta|_{\infty, \Omega^{T}}, T\right)\left[\|F+\widetilde{F}\|_{2, \Omega^{T}}\right. \\
& +\|G+\widetilde{G}\|_{3-1 / 2, S^{T}}+\left|D_{\xi, t}^{2} G+D_{\xi, t}^{2} \widetilde{G}\right|_{1 / 4, S^{T}} \\
& \left.+\phi_{2}\left(|\eta|_{2,0, \infty, \Omega^{T}},\|\eta\|_{C^{\alpha}\left(\Omega^{T}\right)}\right)\|u\|_{2, \Omega^{T}}\right] \\
& +\phi_{3}\left(|1 / \eta|_{\infty, \Omega^{T}}, T\right)|u(0)|_{3,0, \Omega}
\end{aligned}
$$

First we estimate $\|\widetilde{F}\|_{2, \Omega^{T}}$. By the form of the operator $\nabla_{w}$ we obtain

$$
\begin{aligned}
& \|\widetilde{F}\|_{2, \Omega^{T}} \\
& \leq c\left\|\left(\xi_{x} \nabla_{\xi}\right)^{2} u-\nabla_{\xi}^{2} u\right\|_{2, \Omega^{T}}+c\left\|\xi_{x} \nabla_{\xi}\left(\xi_{x} \nabla_{\xi} \cdot u\right)-\nabla_{\xi} \nabla_{\xi} \cdot u\right\|_{2, \Omega^{T}} \\
& \leq c\left\|\left(\xi_{x} \nabla_{\xi}\right)^{2} u-\nabla_{\xi}^{2} u\right\|_{2, \Omega^{T}} \\
& \quad+c\left\|\xi_{x} \nabla_{\xi}\left(\xi_{x}\right) \nabla_{\xi} \cdot u\right\|_{2, \Omega^{T}}+c\left\|\left(\xi_{x}^{2}-\mathbf{1}\right) D_{\xi}^{2} u\right\|_{2, \Omega^{T}} \\
& \leq c\left[\left\|f_{1} \int_{0}^{t} D_{\xi}^{2} w d \tau D_{\xi}^{1} u\right\|_{2, \Omega^{T}}+\left\|f_{2} \int_{0}^{t} D_{\xi}^{1} w d \tau D_{\xi}^{2} u\right\|_{2, \Omega^{T}}\right],
\end{aligned}
$$

where $f_{i}=f_{i}\left(\mathbf{1}+\int_{0}^{t} w_{\xi} d \tau\right)(i=1,2)$ are smooth functions of their arguments; $\xi_{x}=x_{\xi}^{-1}=\widetilde{x}_{\xi} / \operatorname{det}\left\{x_{\xi}\right\}, \widetilde{x}_{\xi}$ is the matrix of algebraic complements of $\left\{x_{\xi}\right\}$ and $x_{\xi}=\mathbf{1}+\int_{0}^{t} w_{\xi} d \tau$. The products on the right-hand side of (3.52) (and below) are in the sense of (2.2).

Now, applying Hölder and Minkowski inequalities and Lemmas 2.1-2.2 we get

$$
\begin{array}{rl}
\left\|f_{1} \int_{0}^{t} D_{\xi}^{2} w d \tau u_{\xi}\right\|_{2, \Omega^{T}}+\left\|f_{2} \int_{0}^{t} w_{\xi} d \tau D_{\xi}^{2} u\right\|_{2, \Omega^{T}} \\
\leq c & c T\|w\|_{4, \Omega^{T}}^{2}\|u\|_{4, \Omega^{T}}+T^{3 / 2}\|w\|_{4, \Omega^{T}}^{3}\|u\|_{4, \Omega^{T}} \\
& +T^{1 / 2}\|w\|_{4, \Omega^{T}}\|u\|_{4, \Omega^{T}} \\
& +T^{1 / 2}\|w\|_{4, \Omega^{T}}^{2}\left(\|u\|_{4, \Omega^{T}}+\|u(0)\|_{3, \Omega}\right) \\
& \left.+T^{1 / 2}\left(\|w\|_{4, \Omega^{T}}+\|w(0)\|_{3, \Omega}\right)\left(\|u\|_{4, \Omega^{T}}+\|u(0)\|_{3, \Omega}\right)\right],
\end{array}
$$

where $c=c\left(\int_{0}^{T}\left|w_{\xi}\right|_{\infty, \Omega} d t\right)$.

Next consider

$$
\begin{aligned}
\|\widetilde{G}\|_{3-1 / 2, S^{T}} & \leq c\left\|f_{3} \int_{0}^{t} w_{\xi} d \tau u_{\xi} \widetilde{\phi}_{\xi}\right\|_{3-1 / 2, S^{T}} \\
& \leq c\left(\sum_{|\alpha|+2 i \leq 2}\left|D_{\xi}^{\alpha} \partial_{t}^{i}\left(f_{3} \int_{0}^{t} w_{\xi} d \tau u_{\xi} \widetilde{\phi}_{\xi}\right)\right|_{2, S^{T}}\right.
\end{aligned}
$$




$$
\begin{aligned}
& +\sum_{|\alpha|+2 i=2}\left[D_{\xi}^{\alpha} \partial_{t}^{i}\left(f_{3} \int_{0}^{t} w_{\xi} d \tau u_{\xi} \widetilde{\phi}_{\xi}\right)\right]_{1 / 2, S^{T}, \xi} \\
& \left.+\sum_{|\alpha|+2 i=2}\left[D_{\xi}^{\alpha} \partial_{t}^{i}\left(f_{3} \int_{0}^{t} w_{\xi} d \tau u_{\xi} \widetilde{\phi}_{\xi}\right)\right]_{1 / 4, S^{T}, t}\right),
\end{aligned}
$$

where $f_{3}=f_{3}\left(\mathbf{1}+\int_{0}^{t} w_{\xi} d \tau\right)$ is a smooth function of its arguments.

By the Hölder and Minkowski inequalities, Lemma 2.1 (inequality (2.5)) and Lemma 2.2 we have

$$
\begin{aligned}
\text { (3.55) } & \sum_{|\alpha|+2 i \leq 2}\left|D_{\xi}^{\alpha} \partial_{t}^{i}\left(f_{3} \int_{0}^{t} w_{\xi} d \tau u_{\xi} \widetilde{\phi}_{\xi}\right)\right|_{2, S^{T}} \\
\leq & c\left[T^{1 / 2}\|w\|_{4, \Omega^{T}}\|u\|_{4, \Omega^{T}}+T\|w\|_{4, \Omega^{T}}^{2}\|u\|_{4, \Omega^{T}}+T^{3 / 2}\|w\|_{4, \Omega^{T}}^{3}\|u\|_{4, \Omega^{T}}\right. \\
& +T^{1 / 2}\|w\|_{4, \Omega^{T}}^{2}\left(\|u\|_{4, \Omega^{T}}+|u(0)|_{3,0, \Omega}\right) \\
& \left.+T^{1 / 2}\left(\|w\|_{4, \Omega^{T}}+|w(0)|_{3,0, \Omega}\right)\left(\|u\|_{4, \Omega^{T}}+|u(0)|_{3,0, \Omega}\right)\right] \equiv I,
\end{aligned}
$$

where $c=c\left(\int_{0}^{T}\left|w_{\xi}\right|_{\infty, \Omega} d t\right)$.

Now consider

$$
I_{1} \equiv \sum_{|\alpha|+2 i=2}\left[D_{\xi}^{\alpha} \partial_{t}^{i}\left(f_{3} \int_{0}^{t} w_{\xi} d \tau u_{\xi} \widetilde{\phi}_{\xi}\right)\right]_{1 / 2, S^{T}, \xi} .
$$

We shall only estimate some terms of $I_{1}$. For example we have

$$
\begin{aligned}
& I_{2} \equiv\left\{\int_{0}^{T} \int_{S} \int_{S} \frac{\mid\left[f_{3}^{\prime \prime}\left(\int_{0}^{t} D_{\xi}^{2} w d \tau\right)^{2} \int_{0}^{t} w_{\xi} d \tau u_{\xi} \widetilde{\phi}_{\xi}\right](\xi)}{\ldots} \cdots\right. \\
& \leq c\left\{\int_{0}^{T} \int_{S} \int_{S} \frac{\left.\mid\left[f_{3}^{\prime \prime}\left(\int_{0}^{t} w_{\xi} d \tau\right)\right](\xi)-\left[f_{3}^{\prime \prime} \int_{0}^{t} w_{\xi^{\prime}} d \tau\right)\right]\left.\left(\xi^{\prime}\right)\right|^{2}}{\left|\xi-\xi^{\prime}\right|^{3}}\right. \\
&\left.\times\left|\int_{0}^{t} D_{\xi}^{2} w d \tau\right|^{2} \mid \int_{0}^{t} w_{\xi^{\prime}} d \tau u_{\xi^{\prime}} \widetilde{\phi}_{\xi^{\prime}}\right]\left.\left(\xi^{\prime}\right)\right|^{2} \\
&\left.\left|w_{\xi} d \tau\right|^{2}\left|u_{\xi}\right|^{2}\left|\widetilde{\phi}_{\xi}\right|^{2} d \xi d \xi^{\prime} d t\right\}^{1 / 2} \\
&+c\left\{\int_{0}^{T} \int_{S} \int_{S} \frac{\left|\int_{0}^{t}\left[D_{\xi}^{2} w(\xi)-D_{\xi^{\prime}}^{2} w\left(\xi^{\prime}\right)\right] d \tau\right|^{2}}{\left|\xi-\xi^{\prime}\right|^{3}}\right. \\
&\left.\times\left|\int_{0}^{t} D_{\xi}^{2} w d \tau\right|^{2}\left|\int_{0}^{t} w_{\xi} d \tau\right|^{2}\left|u_{\xi}\right|^{2}\left|\widetilde{\phi}_{\xi}\right|^{2} d \xi d \xi^{\prime} d t\right\}^{1 / 2}
\end{aligned}
$$




$$
\begin{aligned}
& +c\left\{\int_{0}^{T} \int_{S} \int_{S} \frac{\left|\int_{0}^{t}\left[w_{\xi}(\xi)-w_{\xi^{\prime}}\left(\xi^{\prime}\right)\right] d \tau\right|^{2}}{\left|\xi-\xi^{\prime}\right|^{3}}\left|\int_{0}^{t} D_{\xi}^{2} w d \tau\right|^{4}\left|u_{\xi}\right|^{2}\left|\widetilde{\phi}_{\xi}\right|^{2} d \xi d \xi^{\prime} d t\right\}^{1 / 2} \\
& +c\left\{\int_{0}^{T} \int_{S} \int_{S} \frac{\left|u_{\xi}(\xi)-u_{\xi^{\prime}}\left(\xi^{\prime}\right)\right|^{2}}{\left|\xi-\xi^{\prime}\right|^{3}}\left|\int_{0}^{t} D_{\xi}^{2} w d \tau\right|^{4}\left|\int_{0}^{t} w_{\xi} d \tau\right|^{2}\left|\widetilde{\phi}_{\xi}\right|^{2} d \xi d \xi^{\prime} d t\right\}^{1 / 2} \\
& +c\left\{\int_{0}^{T} \int_{S} \int_{S} \frac{\left|\widetilde{\phi}_{\xi}(\xi)-\widetilde{\phi}_{\xi^{\prime}}\left(\xi^{\prime}\right)\right|^{2}}{\left|\xi-\xi^{\prime}\right|^{3}}\left|\int_{0}^{t} D_{\xi}^{2} w d \tau\right|^{4}\left|\int_{0}^{t} w_{\xi} d \tau\right|^{2}\left|u_{\xi}\right|^{2} d \xi d \xi^{\prime} d t\right\}^{1 / 2} .
\end{aligned}
$$

Using the Taylor formula we get

$$
\begin{aligned}
(3.56) \quad & I_{2} \leq c\left\{\int_{0} \int_{S} \int_{S} \frac{\left|\int_{0}^{t} D_{\xi}^{2} w(\widetilde{\xi}) d \tau\right|^{2}}{\left|\xi-\xi^{\prime}\right|}\right. \\
\times & \left.\left|\int_{0}^{t} D_{\xi}^{2} w d \tau\right|^{4}\left|\int_{0}^{t} w_{\xi} d \tau\right|^{2}\left|u_{\xi}\right|^{2}\left|\widetilde{\phi}_{\xi}\right|^{2} d \xi d \xi^{\prime} d t\right\}^{1 / 2} \\
+ & c\left\{\int_{0}^{T} \int_{S} \int_{S} \frac{\left|\int_{0}^{t}\left[D_{\xi}^{2} w(\xi)-D_{\xi^{\prime}}^{2} w\left(\xi^{\prime}\right)\right] d \tau\right|^{2}}{\left|\xi-\xi^{\prime}\right|^{3}}\right. \\
\times & \left.\left|\int_{0}^{t} D_{\xi}^{2} w d \tau\right|^{2}\left|\int_{0}^{t} w_{\xi} d \tau\right|^{2}\left|u_{\xi}\right|^{2}\left|\widetilde{\phi}_{\xi}\right|^{2} d \xi d \xi^{\prime} d t\right\}^{1 / 2} \\
+ & c\left\{\int_{0}^{T} \int_{S} \int_{S} \frac{\left|\int_{0}^{t} D_{\xi}^{2} w(\widetilde{\xi}) d \tau\right|^{2}}{\left|\xi-\xi^{\prime}\right|}\left|\int_{0}^{t} D_{\xi}^{2} w d \tau\right|^{4}\left|u_{\xi}\right|^{2}\left|\widetilde{\phi}_{\xi}\right|^{2} d \xi d \xi^{\prime} d t\right\}^{1 / 2} \\
+ & c\left\{\int_{0}^{T} \int_{S} \int_{S} \frac{\left|D_{\xi}^{2} u(\widetilde{\xi})\right|^{2}}{\left|\xi-\xi^{\prime}\right|}\left|\int_{0}^{t} D_{\xi}^{2} w d \tau\right|^{4}\left|\int_{0}^{t} w_{\xi} d \tau\right|^{2}\left|\widetilde{\phi}_{\xi}\right|^{2} d \xi d \xi^{\prime} d t\right\}^{1 / 2} \\
+ & c\left\{\int_{0}^{T} \int_{S} \int_{S} \frac{\left|D_{\xi}^{2} \widetilde{\phi}(\widetilde{\xi})\right|^{2}}{\left|\xi-\xi^{\prime}\right|}\left|\int_{0}^{t} D_{\xi}^{2} w d \tau\right|^{4}\left|\int_{0}^{t} w_{\xi} d \tau\right|^{2}\left|u_{\xi}\right|^{2} d \xi d \xi^{\prime} d t\right\}^{1 / 2} \\
\equiv I_{2}^{1}+I_{2}^{2}+I_{2}^{3}+I_{2}^{4}+I_{2}^{5}, & \theta \xi+(1-\theta) \xi, 0<\theta<1 .
\end{aligned}
$$

Obviously, we have

$$
I_{2}^{1} \leq c T^{2}\|w\|_{4, \Omega^{T}}^{4}\|u\|_{4, \Omega^{T}} .
$$

For $I_{2}^{2}$, we obtain

$$
I_{2}^{2} \leq c\left\{\int_{0}^{T} \sup _{S}\left|\int_{0}^{t} D_{\xi}^{2} w d \tau\right|^{2} \sup _{S}\left|\int_{0}^{t} w_{\xi} d \tau\right|^{2} \sup _{S}\left|u_{\xi}\right|^{2} \sup _{S}\left|\widetilde{\phi}_{\xi}\right|^{2}\right.
$$




$$
\begin{aligned}
& \left.\times \int_{S} \int_{S} \frac{\left|\int_{0}^{t}\left[D_{\xi}^{2} w(\xi)-D_{\xi^{\prime}}^{2} w\left(\xi^{\prime}\right)\right] d \tau\right|^{2}}{\left|\xi-\xi^{\prime}\right|^{3}} d \xi d \xi^{\prime} d t\right\}^{1 / 2} \\
\leq & c T^{1 / 2}\|w\|_{4, \Omega^{T}} T^{1 / 2}\|w\|_{4, \Omega^{T}} T^{1 / 2} \\
& \times\left\{\int_{0}^{T} \int_{S} \int_{S} \frac{\left|D_{\xi}^{2} w(\xi)-D_{\xi^{\prime}}^{2} w\left(\xi^{\prime}\right)\right|^{2}}{\left|\xi-\xi^{\prime}\right|^{3}} d \xi d \xi^{\prime} d t\right\}^{1 / 2}\|u\|_{4, \Omega^{T}} \\
\leq & c T^{3 / 2}\|w\|_{4, \Omega^{T}}^{3}\|u\|_{4, \Omega^{T}},
\end{aligned}
$$

where we have used the fact that $D_{\xi}^{2} w \in W_{2}^{1 / 2,1 / 4}\left(S^{T}\right)$ and Lemma 2.1 (inequality (2.5)).

Next we estimate

$$
\begin{aligned}
I_{2}^{3} \leq & c\left\{\int_{0}^{T} \sup _{S}\left|\int_{0}^{t} D_{\xi}^{2} w d \tau\right|^{4} \sup _{S}\left|u_{\xi}\right|^{2} \sup _{S}\left|\widetilde{\phi}_{\xi}\right|^{2}\right. \\
& \left.\times \sup _{S}\left|\int_{0}^{t} D_{\xi}^{2} w(\widetilde{\xi}) d \tau\right|^{2}\left(\int_{S} \int_{S} \frac{d \xi d \xi^{\prime}}{\left|\xi-\xi^{\prime}\right|}\right) d t\right\}^{1 / 2} \\
\leq & c T^{3 / 2}\|w\|_{4, \Omega^{T}}^{3}\|u\|_{4, \Omega^{T}} .
\end{aligned}
$$

The other terms both of (3.56) and of (3.55) can be estimated in the same way.

Therefore, by the above considerations

$$
\sum_{|\alpha|+2 i=2}\left[D_{\xi}^{\alpha} \partial_{t}^{i}\left(f_{3} \int_{0}^{t} w_{\xi} d \tau u_{\xi} \widetilde{\phi}_{\xi}\right)\right]_{1 / 2, S^{T}, \xi} \leq\left(1+T\|w\|_{4, \Omega^{T}}\right) I,
$$

where $I$ is defined in (3.55).

Consider now

$$
\begin{aligned}
& \sum_{|\alpha|+2 i=2}\left[D _ { \xi } ^ { \alpha } \partial _ { t } ^ { i } \left(f_{3} \int_{0}^{t} w_{\xi} d \tau\right.\right.\left.\left.u_{\xi} \widetilde{\phi}_{\xi}\right)\right]_{1 / 4, S^{T}, t} \\
& \leq c\left[\int_{S} \int_{0}^{T} \int_{0}^{T} \frac{A\left(\xi, t, t^{\prime}\right)}{\left|t-t^{\prime}\right|^{3 / 2}} d \xi d t d t^{\prime}\right]^{1 / 2} \equiv I_{3}
\end{aligned}
$$

where

$$
\begin{aligned}
A\left(\xi, t, t^{\prime}\right) \equiv & \mid D_{\xi, t}^{2}\left(f_{3} \int_{0}^{t} w_{\xi} d \tau u_{\xi} \widetilde{\phi}_{\xi}\right)(t) \\
& -\left.D_{\xi, t^{\prime}}^{2}\left(f_{3} \int_{0}^{t} w_{\xi} d \tau u_{\xi} \widetilde{\phi}_{\xi}\right)\left(t^{\prime}\right)\right|^{2} .
\end{aligned}
$$


As before we only estimate some terms of $I_{3}$. Set

$$
\begin{aligned}
B\left(\xi, t, t^{\prime}\right) \equiv & \mid\left(f_{3}^{\prime \prime}\left(\int_{0}^{t} D_{\xi}^{2} w d \tau\right)^{2} \int_{0}^{t} w_{\xi} d \tau u_{\xi} \widetilde{\phi}_{\xi}\right)(t) \\
& -\left.\left(f_{3}^{\prime \prime}\left(\int_{0}^{t} D_{\xi}^{2} w d \tau\right)^{2} \int_{0}^{t} w_{\xi} d \tau u_{\xi} \widetilde{\phi}_{\xi}\right)\left(t^{\prime}\right)\right|^{2} .
\end{aligned}
$$

We have

$$
\begin{aligned}
& \text { (3.57) } I_{5} \equiv\left[\int_{S} \int_{0}^{T} \int_{0}^{T} \frac{B\left(\xi, t, t^{\prime}\right)}{\left|t-t^{\prime}\right|^{3 / 2}} d \xi d t d t^{\prime}\right]^{1 / 2} \\
& \leq c\left(\int_{S} \int_{0}^{T} \int_{0}^{T} \frac{\left|f_{3}^{\prime \prime}\left(\int_{0}^{t} w_{\xi} d \tau\right)-f_{3}^{\prime \prime}\left(\int_{0}^{t^{\prime}} w_{\xi} d \tau\right)\right|^{2}}{\left|t-t^{\prime}\right|^{3 / 2}}\right. \\
& \left.\quad \times\left|\int_{0}^{t} D_{\xi}^{2} w d \tau\right|^{4}\left|\int_{0}^{t} w_{\xi} d \tau\right|^{2}\left|u_{\xi}\right|^{2}\left|\widetilde{\phi}_{\xi}\right|^{2} d \xi d t d t^{\prime}\right)^{1 / 2} \\
& +c\left(\int_{S} \int_{0}^{T} \int_{0}^{T} \frac{\left|\int_{0}^{t} D_{\xi}^{2} w d \tau-\int_{0}^{t^{\prime}} D_{\xi}^{2} w d \tau\right|^{2}}{\left|t-t^{\prime}\right|^{3 / 2}}\right. \\
& \left.\quad \times\left|\int_{0}^{t} D_{\xi}^{2} w d \tau\right|^{2}\left|\int_{0}^{t} w_{\xi} d \tau\right|^{2}\left|u_{\xi}\right|^{2}\left|\widetilde{\phi}_{\xi}\right|^{2} d \xi d t d t^{\prime}\right)^{1 / 2} \\
& +c\left(\int_{S} \int_{0}^{T} \int_{0}^{T} \frac{\left|u_{\xi}(t)-u_{\xi}\left(t^{\prime}\right)\right|^{2}}{\left.\left.\left|t-t^{\prime}\right|^{3 / 2} \int_{0}^{t} D_{\xi}^{2} w d \tau\right|^{4}\left|\int_{0}^{t} w_{\xi} d \tau\right|^{2}\left|\widetilde{\phi}_{\xi}\right|^{2} d \xi d t d t^{\prime}\right)^{1 / 2}}\right. \\
& \quad+c\left(\int_{S} \int_{0}^{T} \int_{0}^{T} \frac{\left|\int_{0}^{t} w_{\xi} d \tau-\int_{0}^{t^{\prime}} w_{\xi} d \tau\right|^{2}}{\left|t-t^{\prime}\right|^{3 / 2}}\left|\int_{0}^{t} D_{\xi}^{2} w d \tau\right|^{4}\left|u_{\xi}\right|^{2}\left|\widetilde{\phi}_{\xi}\right|^{2} d \xi d t d t^{\prime}\right)^{1 / 2} \\
& \equiv I_{4}^{1}+I_{4}^{2}+I_{4}^{3}+I_{4}^{4} .
\end{aligned}
$$

Using the Taylor formula we obtain

$$
\begin{aligned}
I_{4}^{2} \equiv & c\left(\int_{S} \int_{0}^{T} \int_{0}^{T} \frac{\left|\int_{0}^{t} D_{\xi}^{2} w d \tau-\int_{0}^{t^{\prime}} D_{\xi}^{2} w d \tau\right|^{2}}{\left|t-t^{\prime}\right|^{3 / 2}}\right. \\
& \left.\times\left|\int_{0}^{t} D_{\xi}^{2} w d \tau\right|^{2}\left|\int_{0}^{t} w_{\xi} d \tau\right|^{2}\left|u_{\xi}\right|^{2}\left|\widetilde{\phi}_{\xi}\right|^{2} d \xi d t d t^{\prime}\right)^{1 / 2} \\
\leq & c\left(\int_{0}^{T} \int_{0}^{T}\left|t-t^{\prime}\right|^{1 / 2}\left(\int_{S}\left|D_{\xi}^{2} w(\widetilde{t})\right|^{2} d \xi\right)\right.
\end{aligned}
$$




$$
\begin{aligned}
& \left.\times \sup _{S}\left|\int_{0}^{t} D_{\xi}^{2} w d \tau\right|^{2} \sup _{S}\left|\int_{0}^{t} w_{\xi} d \tau\right|^{2} \sup _{S}\left|u_{\xi}\right|^{2} \sup _{S}\left|\widetilde{\phi}_{\xi}\right|^{2} d t d t^{\prime}\right)^{1 / 2} \\
\leq & c T\|w\|_{4, \Omega^{T}}^{2} \sup _{\tilde{t}}\|w(\widetilde{t})\|_{3, \Omega}^{2}\left(\int_{0}^{T} \int_{0}^{T} T^{1 / 2} \sup _{S}\left|u_{\xi}\right|^{2} d t d t^{\prime}\right)^{1 / 2} \\
\leq & c T^{5 / 4}\|w\|_{4, \Omega^{T}}^{2}\left(\|w\|_{4, \Omega^{T}}+|w(0)|_{3,0, \Omega}\right)\|u\|_{4, \Omega^{T}}
\end{aligned}
$$

where $\widetilde{t}=\theta t+(1-\theta) t^{\prime}, 0<\theta<1$.

In the same way the other terms both of (3.57) and of

$$
\sum_{|\alpha|+2 i=2}\left[D_{\xi}^{\alpha} \partial_{t}^{i}\left(f_{3} \int_{0}^{t} w_{\xi} d \tau u_{\xi} \widetilde{\phi}_{\xi}\right)\right]_{1 / 4, S^{T}, t}
$$

can be estimated.

Thus, from the above considerations we obtain

$$
\begin{aligned}
\|\widetilde{G}\|_{3-1 / 2, S^{T}} \leq & T^{1 / 2}\left(\|w\|_{4, \Omega^{T}}+|w(0)|_{3,0, \Omega}\right) \\
& \times \phi\left(\int_{0}^{T}|w|_{\infty, \Omega} d t,\|w\|_{4, \Omega^{T}},|w(0)|_{3,0, \Omega}, T\right) \\
& \times\left(\|u\|_{4, \Omega^{T}}+|u(0)|_{3,0, \Omega}\right),
\end{aligned}
$$

where $\phi$ is a nonnegative nondecreasing continuous function of its arguments.

It remains to estimate $\left|D_{\xi, t}^{2} \widetilde{G}\right|_{1 / 4, S^{T}}$. We get

$$
\begin{aligned}
& \left|D_{\xi, t}^{2} \widetilde{G}\right|_{1 / 4, S^{T}}=\left(\int_{0}^{T} \frac{\left|D_{\xi, t}^{2}\left(f_{3} \int_{0}^{t} w_{\xi} d \tau u_{\xi} \widetilde{\phi}_{\xi}\right)\right|_{2, S^{T}}^{2}}{t^{1 / 2}} d t\right)^{1 / 2} \\
& \leq c\left[T^{1 / 4}\|w\|_{4, \Omega^{T}}\|u\|_{4, \Omega^{T}}+T^{3 / 4}\|w\|_{4, \Omega^{T}}\|u\|_{4, \Omega^{T}}\right. \\
& \quad+T^{5 / 4}\|w\|_{4, \Omega^{T}}^{3}\|u\|_{4, \Omega^{T}}+T^{1 / 4}\|w\|_{4, \Omega^{T}}\left(\|u\|_{4, \Omega^{T}}+|u(0)|_{3,0, \Omega}\right) \\
& \left.\quad+T^{1 / 4}\left(\|w\|_{4, \Omega^{T}}+|w(0)|_{3,0, \Omega}\right)\left(\|u\|_{4, \Omega^{T}}+|u(0)|_{3,0, \Omega}\right)\right] .
\end{aligned}
$$

By (3.51)-(3.59) we get

$$
\begin{aligned}
\|u\|_{4, \Omega^{T}} \leq & \phi_{1}\left(|1 / \eta|_{\infty, \Omega^{T}},|\eta|_{\infty, \Omega^{T}}, T\right)\left[\|F\|_{2, \Omega^{T}}+\|G\|_{3-1 / 2, S^{T}}\right. \\
& \left.+\left|D_{\xi, t}^{2} G\right|_{1 / 4, S^{T}}+\phi_{2}\left(|\eta|_{2,0, \infty, \Omega^{T}},\|\eta\|_{C^{\alpha}\left(\Omega^{T}\right)}\right)\|u\|_{2, \Omega^{T}}\right] \\
& +\phi_{3}\left(|1 / \eta|_{\infty, \Omega^{T}}, T\right)|u(0)|_{3,0, \Omega}+T^{a}\left(\|w\|_{4, \Omega^{T}}+|w(0)|_{3,0, \Omega}\right) \\
& \times \phi_{7}\left(|1 / \eta|_{\infty, \Omega^{T}},|\eta|_{\infty, \Omega^{T}}, \int_{0}^{T}|w|_{\infty, \Omega} d t,\|w\|_{4, \Omega^{T}},|w(0)|_{3,0, \Omega}, T\right) \\
& \times\left(\|u\|_{4, \Omega^{T}}+|u(0)|_{3,0, \Omega}\right),
\end{aligned}
$$

where $a>0$ is a constant. 
Therefore if $\delta>0$ is sufficiently small (3.49) follows from (3.48). To prove the existence and uniqueness of solutions to problem (3.46) we use the method of successive approximations. Put $u_{m}$ in the right-hand side of (3.50) in place of $u$ and replace $u$ by $u_{m+1}$ in the left-hand side of (3.50). Then by the contraction theorem we have the existence of solutions of problem (3.46) for sufficiently small $T$. Therefore the theorem is proved.

In the same way the following theorem can be proved:

TheOREM 3.6. Assume that

$S \in W_{2}^{4-1 / 2}, K \in W_{2}^{4,2}\left(\Omega^{T}\right), \widetilde{\gamma} \in W_{2}^{3-1 / 2,3 / 2-1 / 4}\left(S^{T}\right)$,

$\mid D_{\xi, t}^{2} \widetilde{\gamma} \mathbf{|}_{1 / 4, S^{T}}<\infty, \gamma_{0} \in W_{2}^{3}(\Omega), \eta \in L_{\infty}\left(0, T ; \Gamma_{0}^{2,1}(\Omega)\right)$,

$1 / \eta \in L_{\infty}\left(\Omega^{T}\right), \eta>0, \eta \in C^{\alpha}\left(\Omega^{T}\right)(\alpha \in(0,1 / 2))$,

$\beta \in W_{2}^{4,2}\left(\Omega^{T}\right), 1 / \beta \in L_{\infty}\left(\Omega^{T}\right), \beta>0, c_{v} \in C^{2}\left(\mathbb{R}_{+}^{2}\right), c_{v}>0$,

$w \in W_{2}^{4,2}\left(\Omega^{T}\right) \cap C^{0}\left(0, T ; \Gamma_{0}^{3,3 / 2}(\Omega)\right), T<\infty$.

Moreover, let the compatibility condition (3.8) be satisfied. Assume that $T$ is so small that

$$
\begin{aligned}
T^{a}\left(\|w\|_{4, \Omega^{T}}\right. & \left.+|w(0)|_{3,0, \Omega}\right) \phi_{8}\left(\int_{0}^{T}\left|w_{\xi}\right|_{\infty, \Omega} d t,\left\|c_{v}\right\|_{C^{2}(\bar{V})},\right. \\
& \left\|1 / c_{v}\right\|_{C^{0}(\bar{V})},|1 / \eta|_{\infty, \Omega^{T}},|\eta|_{\infty, \Omega^{T}},\|\beta\|_{4, \Omega^{T}},|\beta(0)|_{3,0, \Omega}, \\
& \left.\|w\|_{4, \Omega^{T}},|w(0)|_{3,0, \Omega}, T\right) \leq \delta
\end{aligned}
$$

where $\phi_{8}$ is a nonnegative nondecreasing continuous function of its arguments, $a>0$ is a constant and $\delta>0$ is sufficiently small. Then there exists a unique solution $\gamma \in W_{2}^{4,2}\left(\Omega^{T}\right) \cap C^{0}\left(0, T ; \Gamma_{0}^{3,3 / 2}(\Omega)\right)$ to problem (3.47) satisfying the estimate

$$
\begin{aligned}
\|\gamma\|_{4, \Omega^{T}} \leq & \phi_{4}\left(\left\|c_{v}\right\|_{C^{2}(\bar{V})},\left\|1 / c_{v}\right\|_{C^{0}(\bar{V})},|1 / \eta|_{\infty, \Omega^{T}},|\eta|_{\infty, \Omega^{T}}, T\right) \\
& \times\left[\|K\|_{2, \Omega^{T}}+\|\widetilde{\gamma}\|_{3-1 / 2, S^{T}}+\left|D_{\xi, t}^{2} \widetilde{\gamma}\right|_{1 / 4, S^{T}}\right. \\
& +\phi_{5}\left(\left\|c_{v}\right\|_{C^{2}(\bar{V})},\left\|1 / c_{v}\right\|_{C^{0}(\bar{V})},|\eta|_{2,0, \infty, \Omega^{T}}\right. \\
& \left.\left.\|\eta\|_{C^{\alpha}\left(\Omega^{T}\right)},\|\beta\|_{4, \Omega^{T}},|\beta(0)|_{3,0, \Omega}\right)\|\gamma\|_{2, \Omega^{T}}\right] \\
& +\left.\left.\phi_{6}^{\prime}\left(\left\|1 / c_{v}\right\|_{C^{0}(\bar{V})},|1 / \eta|_{\infty, \Omega^{T}}, T\right)\right|_{\gamma}(0)\right|_{3,0, \Omega},
\end{aligned}
$$

where $\phi_{i}(i=4,5)$ are the functions from Theorem 3.4, and $\phi_{6}^{\prime}$ is a nonnegative increasing function.

Now consider the continuity equation $(3.1)_{2}$. Integrating it we have

$$
\eta(\xi, t)=\varrho_{0}(\xi) \exp \left[-\int_{0}^{t} \nabla_{u} \cdot u(\xi, \tau) d \tau\right] .
$$

The following lemma holds (see [15]). 
Lemma 3.3. Let $\varrho_{0} \in W_{2}^{3}(\Omega), u \in C^{0}\left(0, T ; W_{2}^{3}(\Omega)\right) \cap W_{2}^{4,2}\left(\Omega^{T}\right)$. Then the solution (3.62) of the continuity equation satisfies $\eta \in C^{0}\left(0, T ; W_{2}^{3}(\Omega)\right)$, $\eta_{t} \in C^{0}\left(0, T ; W_{2}^{2}(\Omega)\right) \cap L_{2}\left(0, T ; W_{2}^{3}(\Omega)\right), \eta_{t t} \in L_{2}\left(0, T ; W_{2}^{1}(\Omega)\right)$ and the following estimates hold:

$$
\begin{gathered}
\sup _{t}\|\eta\|_{3, \Omega} \leq \widetilde{\psi}_{1}\left(T^{1 / 2}\|u\|_{4, \Omega^{T}}\right)\left(T^{1 / 2}\|u\|_{4, \Omega^{T}}+1\right)\left\|\varrho_{0}\right\|_{3, \Omega}, \\
\left\|\eta_{t}\right\|_{L_{2}\left(0, T ; W_{2}^{3}(\Omega)\right)} \leq \widetilde{\psi}_{2}\left(T^{1 / 2}\|u\|_{4, \Omega^{T}}\right) T^{1 / 2}\|u\|_{4, \Omega^{T}}\left\|\varrho_{0}\right\|_{3, \Omega},
\end{gathered}
$$

$$
\left\|\eta_{t t}\right\|_{L_{2}\left(0, T ; W_{2}^{1}(\Omega)\right)} \leq \widetilde{\psi}_{3}\left(T^{1 / 2}\|u\|_{4, \Omega^{T}}\right)\left(T^{1 / 2}+1\right)\|u\|_{4, \Omega^{T}}\left\|\varrho_{0}\right\|_{3, \Omega},
$$

$$
\sup _{t}\left\|\eta_{t}\right\|_{2, \Omega} \leq \widetilde{\psi}_{4}\left(T^{1 / 2}\|u\|_{4, \Omega^{T}}\right) \sup _{t}\|u\|_{3, \Omega}\left\|\varrho_{0}\right\|_{3, \Omega} \text {, }
$$

$|1 / \eta|_{\infty, \Omega^{T}} \leq \widetilde{\psi}_{5}\left(T^{1 / 2}\|u\|_{4, \Omega^{T}}\right)\left|1 / \varrho_{0}\right|_{\infty, \Omega}$,

$|\eta|_{\infty, \Omega^{T}} \leq \widetilde{\psi}_{6}\left(T^{1 / 2}\|u\|_{4, \Omega^{T}}\right)\left|\varrho_{0}\right|_{\infty, \Omega}$,

$$
\begin{aligned}
\|\eta\|_{C^{\alpha}\left(\Omega^{T}\right)} \leq & \widetilde{\psi}_{7}\left(T^{1 / 2}\|u\|_{4, \Omega^{T}}\right)\left[\widetilde{\psi}_{8}\left(T^{1 / 2}\|u\|_{4, \Omega}\right)\right. \\
& \left.+T^{1-\alpha}\left(\|u\|_{4, \Omega^{T}}+\|u(0)\|_{3, \Omega}\right)\right]\left\|\varrho_{0}\right\|_{C^{\alpha}(\Omega)},
\end{aligned}
$$

where $\widetilde{\psi}_{i}(i=1, \ldots, 8)$ are nonnegative nondecreasing continuous functions.

Now we are able to prove the existence of solution to problem (3.1).

TheOREM 3.7. Let $S \in W_{2}^{4-1 / 2}, f \in C^{2,1}\left(\mathbb{R}^{3} \times[0, T]\right), r \in C^{2,1}\left(\mathbb{R}^{3} \times\right.$ $[0, T]), \widetilde{\vartheta} \in C^{2,1}\left(\mathbb{R}^{3} \times[0, T]\right), v_{0} \in W_{2}^{3}(\Omega), \vartheta_{0} \in W_{2}^{3}(\Omega), 1 / \vartheta_{0} \in L_{\infty}(\Omega)$, $\vartheta_{0}>0, \varrho_{0} \in W_{2}^{3}(\Omega), 1 / \varrho_{0} \in L_{\infty}(\Omega), \varrho_{0}>0, c_{v} \in C^{2}\left(\mathbb{R}^{2}+\right), c_{v}>0$, $p \in C^{3}\left(\mathbb{R}_{+}^{2}\right)$. Moreover, assume that the following compatibility conditions are satisfied:

$$
D_{\xi}^{\alpha}\left(\mathbb{D}_{\xi}\left(v_{0}\right) \cdot \bar{n}_{0}-p\left(\varrho_{0}, \vartheta_{0}\right) \bar{n}_{0}+p_{0} \bar{n}_{0}\right)=0, \quad|\alpha| \leq 1, \text { on } S,
$$

and

$$
D_{\xi}^{\alpha}\left(\bar{n}_{0} \cdot \nabla_{\xi} \vartheta_{0}-\widetilde{\vartheta}(\xi, 0)\right)=0, \quad|\alpha| \leq 1, \text { on } S .
$$

Let $T^{*}>0$ be so small that $0<c_{1}\left(1-C K_{0} T^{*}\right)^{3} \leq \operatorname{det}\left\{x_{\xi}\right\} \leq c_{2}(1+$ $\left.C K_{0} T^{*}\right)^{3}$ (where $x(\xi, t)=\xi+\int_{0}^{t} u_{0}(\xi, t) d \tau$ for $t \leq T^{*}, u_{0}$ is given by (3.74), $K_{0}$ is given by (3.91) and the constant $C=C\left(K_{0}\right)$, which is a nondecreasing continuous function of $K_{0}$, is given by (3.94)). Then there exists $T^{* *}$ with $0<T^{* *} \leq T^{*}$ such that for $T \leq T^{* *}$ there exists a unique solution $(u, \gamma, \eta) \in$ $W_{2}^{4,2}\left(\Omega^{T}\right) \times W_{2}^{4,2}\left(\Omega^{T}\right) \times C^{0}\left(0, T ; \Gamma_{0}^{3,3 / 2}(\Omega)\right)$ of problem $(3.1)$ and

$$
\|u\|_{4, \Omega^{T}}+\|\gamma\|_{4, \Omega^{T}} \leq C K_{0},
$$

$$
\begin{aligned}
\sup _{t}\|\eta\|_{3, \Omega}+\sup _{t}\left\|\eta_{t}\right\|_{2, \Omega}+\left\|\eta_{t}\right\|_{L_{2}\left(0, T ; W_{2}^{3}(\Omega)\right)} & +\left\|\eta_{t t}\right\|_{L_{2}\left(0, T ; W_{2}^{1}(\Omega)\right)} \\
& \leq \Phi_{1}\left(T, T^{a} K_{0}\right)\left\|\varrho_{0}\right\|_{3, \Omega},
\end{aligned}
$$


$|1 / \eta|_{\infty, \Omega^{T}}+|\eta|_{\infty, \Omega^{T}} \leq \Phi_{2}\left(T^{1 / 2} K_{0}\right)\left|1 / \varrho_{0}\right|_{\infty, \Omega}+\Phi_{3}\left(T^{1 / 2} K_{0}\right)\left|\varrho_{0}\right|_{\infty, \Omega}$, where $\Phi_{i}(i=1,2,3)$ are increasing continuous functions of their arguments, $a>0$.

Proof. We prove the existence of solutions of problem (3.1) applying the method of successive approximations. To do this consider the problems

$$
\begin{array}{cc}
\eta_{m} \partial_{t} u_{m+1}-\mu \nabla_{u_{m}}^{2} u_{m+1}-\nu \nabla_{u_{m}} \nabla_{u_{m}} \cdot u_{m+1} & \\
=-\nabla_{u_{m}} p\left(\eta_{m}, \gamma_{m}\right)+\eta_{m} g & \text { in } \Omega^{T}, \\
\mathbb{D}_{u_{m}}\left(u_{m+1}\right) \cdot \bar{n}\left(u_{m}\right)=\left[p\left(\eta_{m}, \gamma_{m}\right)-p_{0}\right] \bar{n}\left(u_{m}\right) & \text { on } S^{T}, \\
\left.u_{m+1}\right|_{t=0}=v_{0} & \text { in } \Omega ; \\
\eta_{m} c_{v}\left(\eta_{m}, \gamma_{m}\right) \partial_{t} \gamma_{m+1}-\kappa \nabla_{u_{m}}^{2} \gamma_{m+1} & \\
=-\gamma_{m} p_{\gamma}\left(\eta_{m}, \gamma_{m}\right) \nabla_{u_{m}} \cdot u_{m} & \\
+\frac{\mu}{2} \sum_{i, j=1}^{3}\left(\xi_{x_{i}} \cdot \nabla_{\xi} u_{m j}+\xi_{x_{j}} \cdot \nabla_{\xi} u_{m i}\right)^{2} & \\
+(\nu-\mu)\left(\nabla_{u_{m}} \cdot u_{m}\right)^{2}+\eta_{m} k & \text { in } \Omega^{T}, \\
\bar{n}\left(u_{m}\right) \cdot \nabla_{u_{m}} \gamma_{m+1}=\widetilde{\gamma} & \text { on } S^{T}, \\
\left.\gamma_{m+1}\right|_{t=0}=\vartheta_{0} & \text { in } \Omega
\end{array}
$$

(where $\left.\widetilde{\gamma}(\xi, t)=\widetilde{\vartheta}\left(X_{u_{m}}(\xi, t), t\right)=\widetilde{\vartheta}\left(\xi+\int_{0}^{t} u_{m} d \tau, t\right)\right)$ and

$$
\begin{array}{ll}
\partial_{t} \eta_{m}+\eta_{m} \nabla_{u_{m}} \cdot u_{m}=0 & \text { in } \Omega^{T}, \\
\left.\eta_{m}\right|_{t=0}=\varrho_{0} & \text { in } \Omega .
\end{array}
$$

For $u_{0}$ we take a function from Lemma 3.1 such that

$$
\left.\partial_{t}^{i} u_{0}\right|_{t=0}=\psi_{i} \quad \text { in } \Omega(i=0,1)
$$

and

$$
\left\|u_{0}\right\|_{4, \Omega^{T}} \leq c\left(\left\|\psi_{0}\right\|_{3, \Omega}+\left\|\psi_{1}\right\|_{1, \Omega}\right),
$$

where $\psi_{i}=\left.\partial_{t}^{i} u\right|_{t=0}$ are calculated from problem (3.1), i.e.

(3.76) $\quad \psi_{0}=v_{0}, \quad \varrho_{0} \psi_{1}-\mu \nabla_{\xi}^{2} v_{0}-\nu \nabla_{\xi} \nabla_{\xi} \cdot v_{0}+\nabla_{\xi} p\left(\varrho_{0}, \vartheta_{0}\right)=\varrho_{0} g(0)$.

Similarly, for $\gamma_{0}$ we take a function from Lemma 3.1 such that

$$
\left.\partial_{t}^{i} \gamma_{0}\right|_{t=0}=\sigma_{i} \quad \text { in } \Omega(i=0,1)
$$

and

$$
\left\|\gamma_{0}\right\|_{4, \Omega^{T}} \leq c\left(\left\|\sigma_{0}\right\|_{3, \Omega}+\left\|\sigma_{1}\right\|_{1, \Omega}\right)
$$


where $\sigma_{i}=\left.\partial_{t}^{i} \gamma\right|_{t=0}(i=0,1)$, i.e.

$$
\begin{aligned}
& \sigma_{0}=\vartheta_{0} \\
& \eta_{0} c_{v}\left(\eta_{0}, \vartheta_{0}\right) \sigma_{1}-\kappa \nabla_{\xi}^{2} \vartheta_{0}=-\vartheta_{0} p_{\gamma}\left(\eta_{0}, \vartheta_{0}\right) \nabla_{\xi} \cdot v_{0} \\
& \quad+\frac{\mu}{2} \sum_{i, j=1}^{3}\left(\partial_{\xi_{i}} v_{0 j}+\partial_{\xi_{j}} v_{0 i}\right)^{2}+(\nu-\mu)\left(\nabla_{\xi} \cdot v_{0}\right)^{2}+\eta_{0} k(0),
\end{aligned}
$$

and $\eta_{0}$ is a solution of the problem

$$
\partial_{t} \eta_{0}+\eta_{0} \nabla_{u_{0}} u_{0}=0,\left.\quad \eta_{0}\right|_{t=0}=\varrho_{0} .
$$

Assume that $u_{m} \in W_{2}^{4,2}\left(\Omega^{T}\right)$ and $\gamma_{m} \in W_{2}^{4,2}\left(\Omega^{T}\right)$. Then by Lemma $3.3,1 / \eta_{m} \in L_{\infty}\left(\Omega^{T}\right), \eta_{m}>0$ and $\eta_{m} \in C^{\alpha}\left(\Omega^{T}\right)$. Next, since $\gamma_{m}(t)=$ $\int_{0}^{t} \partial_{\tau} \gamma_{m}(\tau) d \tau+\gamma_{m}(0)$ we have

$$
\gamma_{m}(t) \geq \vartheta_{0}-T^{1 / 2}\left(\int_{0}^{T}\left|\partial_{\tau} \gamma_{m}\right|^{2} d \tau\right)^{1 / 2} .
$$

Thus, from the assumption that $1 / \vartheta_{0} \in L_{\infty}(\Omega)$ and $\vartheta_{0}>0$ it follows that for $T$ sufficiently small, $1 / \gamma_{m} \in L_{\infty}\left(\Omega^{T}\right)$ and $\gamma_{m}>0$.

Now assume that inequalities (3.48) and (3.60) with $w=u_{m}, \eta=\eta_{m}$ and $\beta=\gamma_{m}$ are satisfied with sufficiently small $\delta$. Then by Theorem 3.5 there exists a unique solution $u_{m+1} \in W_{2}^{4,2}\left(\Omega^{T}\right)$ (where $T=T(\delta)$ is also small) of (3.71) such that

$$
\begin{aligned}
\left\|u_{m+1}\right\|_{4, \Omega^{T}} \leq & \phi_{1}\left(\left|1 / \eta_{m}\right|_{\infty, \Omega^{T}},\left|\eta_{m}\right|_{\infty, \Omega^{T}}, T\right) \\
& \times\left[\left\|\nabla_{u_{m}} p\left(\eta_{m}, \gamma_{m}\right)\right\|_{2, \Omega^{T}}+\left\|\eta_{m} g\right\|_{2, \Omega^{T}}\right. \\
& +\left\|\left[p\left(\eta_{m}, \gamma_{m}\right)-p_{0}\right] \bar{n}\left(u_{m}\right)\right\|_{3-1 / 2, S^{T}} \\
& +\left|D_{\xi, t}^{2}\left\{\left[p\left(\eta_{m}, \gamma_{m}\right)-p_{0}\right] \bar{n}\left(u_{m}\right)\right\}\right|_{1 / 4, S^{T}} \\
& \left.+\phi_{2}\left(\left|\eta_{m}\right|_{2,0, \infty, \Omega^{T}},\left\|\eta_{m}\right\|_{C^{\alpha}\left(\Omega^{T}\right)}\right)\left\|u_{m}\right\|_{2, \Omega^{T}}\right] \\
& +\phi_{3}^{\prime}\left(\left|1 / \eta_{m}\right|_{\infty, \Omega^{T}}, T\right)\left|u_{m}(0)\right|_{3,0, \Omega} .
\end{aligned}
$$

Similarly, by Theorem 3.6 there is a unique solution $\gamma_{m+1} \in W_{2}^{4,2}\left(\Omega^{T}\right)$ $(T=T(\delta))$ of $(3.72)$ such that

$$
\begin{aligned}
& \left\|\gamma_{m+1}\right\|_{4, \Omega^{T}} \\
& \leq \phi_{4}\left(\left\|c_{v}\right\|_{C^{2}\left(\bar{V}_{m}\right)},\left\|1 / c_{v}\right\|_{C^{0}\left(\bar{V}_{m}\right)},\left|1 / \eta_{m}\right|_{\infty, \Omega^{T}},\left|\eta_{m}\right|_{\infty, \Omega^{T}}, T\right) \\
& \quad \times\left[\left\|\gamma_{m} p_{\gamma}\left(\eta_{m}, \gamma_{m}\right) \nabla_{u_{m}} u_{m}\right\|_{2, \Omega^{T}}\right. \\
& \quad+\left\|\left(\nabla_{u_{m}} u_{m}\right)^{2}\right\|_{2, \Omega^{T}}+\left\|\eta_{m} k\right\|_{2, \Omega^{T}}+\|\widetilde{\gamma}\|_{3-1 / 2, S^{T}}+\left|D_{\xi, t}^{2} \widetilde{\gamma}\right|_{1 / 4, S^{T}}
\end{aligned}
$$




$$
\begin{aligned}
& +\phi_{5}\left(\left\|c_{v}\right\|_{C^{2}\left(\bar{V}_{m}\right)},\left\|1 / c_{v}\right\|_{C^{0}\left(\bar{V}_{m}\right)},\left|\eta_{m}\right|_{2,0, \infty, \Omega^{T}},\right. \\
& \left.\left.\left\|\eta_{m}\right\|_{C^{\alpha}\left(\Omega^{T}\right)},\left\|\gamma_{m}\right\|_{4, \Omega^{T}},\left|\gamma_{m}(0)\right|_{3,0, \Omega}\right)\left\|\gamma_{m}\right\|_{2, \Omega^{T}}\right] \\
& +\phi_{6}^{\prime}\left(\left\|1 / c_{v}\right\|_{C^{0}\left(\bar{V}_{m}\right)},\left|1 / \eta_{m}\right|_{\infty, \Omega^{T}}, T\right)\left|\gamma_{m}(0)\right|_{3,0, \Omega},
\end{aligned}
$$

where $V_{m} \subset \mathbb{R}_{+}^{2}$ is a bounded domain such that $\left(\eta_{m}(\xi, t), \gamma_{m}(\xi, t)\right) \in V_{m}$ for any $(\xi, t) \in \Omega^{T}$.

In order to estimate the terms on the right-hand sides of (3.81) and (3.82) we use the same methods as in Theorem 3.5. Thus, we get

(3.83) $\left\|\nabla_{u_{m}} p\left(\eta_{m}, \gamma_{m}\right)\right\|_{2, \Omega^{T}}$

$=\left\|\xi_{x} \nabla_{\xi} p\left(\eta_{m}, \gamma_{m}\right)\right\|_{2, \Omega^{T}} \leq\left\|\xi_{x}\left(p_{\eta} D_{\xi}^{1} \eta_{m}+p_{\gamma} D_{\xi}^{1} \gamma_{m}\right)\right\|_{2, \Omega^{T}}$

$\leq \sum_{|\alpha|+2 i \leq 2}\left|D_{\xi}^{\alpha} \partial_{t}^{i}\left[\widetilde{f}_{1}\left(p_{\eta} D_{\xi}^{1} \eta_{m}+p_{\gamma} D_{\xi}^{1} \gamma_{m}\right)\right]\right|_{2, \Omega^{T}}$

$\leq c\left\|\widetilde{f}_{1}\right\|_{C^{2}\left(\bar{G}_{m}\right)}\|p\|_{C^{2}\left(\bar{V}_{m}\right)} \alpha_{1}\left(T^{a}\left(\sup _{t}\left\|\partial_{t} \eta_{m}\right\|_{1, \Omega}+\sup _{t}\left\|\eta_{m}\right\|_{3, \Omega}\right)\right.$,

$\left.T^{a}\left(\left\|u_{m}\right\|_{4, \Omega^{T}}+\sup _{t}\left|u_{m}(t)\right|_{3,0, \Omega}+\left\|\gamma_{m}\right\|_{4, \Omega^{T}}+\sup _{t}\left|\gamma_{m}(t)\right|_{3,0, \Omega}\right), T^{a_{1}}\right)$,

(3.84) $\quad\left\|\gamma_{m} p_{\gamma}\left(\eta_{m}, \gamma_{m}\right) \nabla_{u_{m}} \cdot u_{m}\right\|_{2, \Omega^{T}}=\left\|\gamma_{m} p_{\gamma}\left(\eta_{m}, \gamma_{m}\right) \tilde{f}_{1} \cdot D_{\xi}^{1} u_{m}\right\|_{2, \Omega^{T}}$

$\leq c\left\|\widetilde{f}_{1}\right\|_{C^{2}\left(\bar{G}_{m}\right)}\|p\|_{C^{3}\left(\bar{V}_{m}\right)} \alpha_{2}\left(T^{a}\left(\sup _{t}\left\|\partial_{t} \eta_{m}\right\|_{1, \Omega}+\sup _{t}\left\|\eta_{m}\right\|_{3, \Omega}\right)\right.$,

$$
T^{a}\left(\left\|u_{m}\right\|_{4, \Omega^{T}}+\sup _{t}\left|u_{m}(t)\right|_{3,0, \Omega}+\left\|\gamma_{m}\right\|_{4, \Omega^{T}}+\sup _{t}\left|\gamma_{m}(t)\right|_{3,0, \Omega}, T^{a_{2}}\right)
$$

and

$$
\begin{aligned}
& \left\|\left(\nabla_{u_{m}} \cdot u_{m}\right)^{2}\right\|_{2, \Omega^{T}}+\left\|\sum_{i, j=1}^{3}\left(\xi_{x_{i}} \cdot \nabla_{\xi} u_{m j}+\xi_{x_{j}} \cdot \nabla_{\xi} u_{m i}\right)^{2}\right\|_{2, \Omega^{T}} \\
& \quad \leq\left\|\left(\widetilde{f}_{2} \nabla_{\xi} u_{m}\right)^{2}\right\|_{2, \Omega^{T}} \\
& \leq c\left\|\widetilde{f}_{2}\right\|_{C^{2}\left(\bar{G}_{m}\right)} \alpha_{3}\left(T^{a}\left(\left\|u_{m}\right\|_{4, \Omega^{T}}+\sup _{t}\left|u_{m}(t)\right|_{3,0, \Omega}\right), T^{a_{3}}\right) .
\end{aligned}
$$

In (3.83)-(3.85), $\widetilde{f}_{i}=\widetilde{f}_{i}\left(\int_{0}^{t} D_{\xi}^{1} u_{m} d \tau\right)(i=1,2)$ are smooth functions, $G_{m} \subset \mathbb{R}^{9}$ is a bounded domain such that $\int_{0}^{T} D_{\xi}^{1} u_{m} d \tau \in G_{m}$ for any $(\xi, t)$ $\in \Omega^{T}, \alpha_{i}(i=1,2,3)$ are nonnegative increasing continuous functions of their arguments which are polynomials such that $\alpha_{i}(0,0,0)=0, a>0$ and $a_{i}>0(i=1,2,3)$ are constants.

Next, we obtain

$$
\begin{array}{r}
\left\|\eta_{m} g\right\|_{2, \Omega^{T}} \leq c\|f\|_{C^{2,1}\left(\bar{Q}_{m}\right)} \alpha_{4}\left(T^{a}\left(\sup _{t}\left\|\eta_{m}\right\|_{3, \Omega}+\sup _{t}\left\|\partial_{t} \eta_{m}\right\|_{1, \Omega}\right),\right. \\
\left.T^{a}\left(\left\|u_{m}\right\|_{4, \Omega^{T}}+\sup _{t}\left|u_{m}(t)\right|_{3,0, \Omega}\right), T^{a_{4}}\right),
\end{array}
$$


and

$$
\begin{array}{r}
\left\|\eta_{m} k\right\|_{2, \Omega^{T}} \leq c\|r\|_{C^{2,1}\left(\bar{Q}_{m}\right)} \alpha_{5}\left(T^{a}\left(\sup _{t}\left\|\eta_{m}\right\|_{3, \Omega}+\sup _{t}\left\|\partial_{t} \eta_{m}\right\|_{1, \Omega}\right),\right. \\
\left.T^{a}\left(\left\|u_{m}\right\|_{4, \Omega^{T}}+\sup _{t}\left|u_{m}(t)\right|_{3,0, \Omega}\right), T^{a_{5}}\right)
\end{array}
$$

(3.88) $\quad\|\widetilde{\gamma}\|_{3-1 / 2, S^{T}}+\mid D_{\xi, t}^{2} \widetilde{\gamma} \mathbf{l}_{1 / 4, S^{T}}$

$$
\leq c\|\widetilde{\vartheta}\|_{C^{2,1}\left(\bar{Q}_{m}\right)} \alpha_{6}\left(T^{a}\left(\left\|u_{m}\right\|_{4, \Omega^{T}}+\sup _{t}\left|u_{m}(t)\right|_{3,0, \Omega}\right), T^{a_{6}}\right),
$$

where $Q_{m} \subset \mathbb{R}^{3} \times[0, T]$ is a bounded domain such that $\left(\xi+\int_{0}^{t} u_{m} d \tau, t\right) \in Q_{m}$ for any $(\xi, t) \in \Omega^{T}, \alpha_{i}(i=4,5,6)$ are nonnegative increasing continuous functions of their arguments which are polynomials such that $\alpha_{i}(0,0,0)=0$, and $a_{i}>0(i=4,5,6)$ are constants.

Finally, we have

$$
\begin{aligned}
& \|[\left.p\left(\eta_{m}, \gamma_{m}\right)-p_{0}\right] \bar{n}\left(u_{m}\right) \|_{3-1 / 2, S^{T}} \\
&+\left|D_{\xi, t}^{2}\left\{\left[p\left(\eta_{m}, \gamma_{m}\right)-p_{0}\right] \bar{n}\left(u_{m}\right)\right\}\right|_{1 / 4, S^{T}} \\
& \leq c\left\{\sum_{|\alpha|+2 i \leq 2}\left|D_{\xi}^{\alpha} \partial_{t}^{i}\left\{\left[p\left(\eta_{m}, \gamma_{m}\right)-p_{0}\right] \widetilde{f}\right\}\right|_{2, S^{T}}\right. \\
&+\sum_{|\alpha|+2 i=2}\left[D_{\xi}^{\alpha} \partial_{t}^{i}\left\{\left[p\left(\eta_{m}, \gamma_{m}\right)-p_{0}\right] \widetilde{f}\right\}\right]_{1 / 2, S^{T}, \xi} \\
&+\sum_{|\alpha|+2 i=2}\left[D_{\xi}^{\alpha} \partial_{t}^{i}\left\{\left[p\left(\eta_{m}, \gamma_{m}\right)-p_{0}\right] \widetilde{f}\right\}\right]_{1 / 4, S^{T}, t} \\
&\left.+\left(\int_{0}^{T} \frac{\left|D_{\xi, t}^{2}\left\{\left[p\left(\eta_{m}, \gamma_{m}\right)-p_{0}\right] \widetilde{f}\right\}\right|_{2, S}^{2}}{t^{1 / 2}} d t\right)^{1 / 2}\right\} \\
& \leq c\|\widetilde{f}\|_{C^{3}\left(\bar{G}_{m}\right)}\|p\|_{C^{3}\left(\bar{G}_{m}\right)} \alpha_{7}\left(T^{a}\left(\sup _{t}\left\|\eta_{m}\right\|_{3, \Omega}+\sup _{t}\left\|\partial_{t} \eta_{m}\right\|_{1, \Omega}\right),\right. \\
&\left.T^{a}\left(\|u\|_{4, \Omega^{T}}+\sup _{t}\left|u_{m}(t)\right|_{3,0, \Omega}+\left\|\gamma_{m}\right\|_{4, \Omega^{T}}+\sup _{t}\left|\gamma_{m}(t)\right|_{3,0, \Omega}\right), T^{a_{7}}\right),
\end{aligned}
$$

where $\alpha_{7}$ has the same properties as $\alpha_{1}$ and $\alpha_{2}, a_{7}>0$ is a constant, and $\widetilde{f}=\widetilde{f}\left(\int_{0}^{t} D_{\xi}^{1} u_{m} d \tau\right)$ is a smooth function.

Let

$$
\begin{aligned}
y_{m}(t)= & \left\|u_{m}\right\|_{4, \Omega^{t}}^{2}+\left\|\gamma_{m}\right\|_{4, \Omega^{t}}^{2}+\sup _{\tau \in(0, t)}\left|u_{m}(t)\right|_{3,0, \Omega}^{2}+\sup _{\tau \in(0, t)}\left|\gamma_{m}(t)\right|_{3,0, \Omega}^{2}, \\
b= & \left\|\varrho_{0}\right\|_{3, \Omega}+\left|1 / \varrho_{0}\right|_{\infty, \Omega}+\left|\varrho_{0}\right|_{\infty, \Omega}+\left|u_{m}(0)\right|_{3,0, \Omega}+\left|\gamma_{m}(0)\right|_{3,0, \Omega}, \\
c_{m}= & \|p\|_{C^{3}\left(\bar{V}_{m}\right)}+\|f\|_{C^{2,1}\left(\bar{Q}_{m}\right)}+\|r\|_{C^{2,1}\left(\bar{Q}_{m}\right)}+\|\widetilde{\vartheta}\|_{C^{2,1}\left(\bar{Q}_{m}\right)}+\left\|c_{v}\right\|_{C^{2}\left(\bar{V}_{m}\right)} \\
& +\left\|1 / c_{v}\right\|_{C^{0}\left(\bar{V}_{m}\right)}+\|\widetilde{f}\|_{C^{2}\left(\bar{G}_{m}\right)}+\sum_{i=1}^{2}\left\|\widetilde{f}_{i}\right\|_{C^{2}\left(\bar{G}_{m}\right)} .
\end{aligned}
$$


Hence, taking into account inequalities (3.81)-(3.89) and using Lemma 3.3 we obtain

$$
\begin{aligned}
y_{m+1}(t) \leq & \beta_{1}\left(t^{a} y_{m}(t), b, c_{m}, t\right) \\
& +\beta_{2}\left(t^{a} y_{m}(t), y_{m}(t), b, c_{m}\right) \int_{0}^{t} y_{m+1}(\tau) d \tau,
\end{aligned}
$$

where $\beta_{i}(i=1,2)$ are nonnegative nondecreasing continuous functions of their arguments and

$$
\beta_{1}\left(0, b, c_{m}, 0\right)=\left[\phi_{3}^{\prime}\left(\widetilde{\psi}_{5}(0)\left|1 / \varrho_{0}\right|_{\infty, \Omega}, T\right)+\phi_{6}^{\prime}\left(c_{m}, \widetilde{\psi}_{5}(0)\left|1 / \varrho_{0}\right|_{\infty, \Omega}, T\right)\right] .
$$

In view of the Gronwall lemma we have

$$
\begin{aligned}
y_{m+1}(t) \leq & \beta_{1}\left(t^{a} y_{m}(t), b, c_{m}, t\right) \\
& \times \exp \left[t \beta_{2}\left(t^{a} y_{m}(t), y_{m}(t), b, c_{m}\right)\right] .
\end{aligned}
$$

Using (3.75) and (3.78) we get

$$
y_{0}(t) \leq c\left(\left\|\psi_{0}\right\|_{3, \Omega}^{2}+\left\|\psi_{1}\right\|_{1, \Omega}^{2}+\left\|\sigma_{0}\right\|_{3, \Omega}^{2}+\left\|\sigma_{1}\right\|_{1, \Omega}^{2}\right)<c b \equiv K_{0} .
$$

Assume that

$$
y_{m}(t) \leq C K_{0} \quad \forall t \leq T^{*}
$$

(where $C>0$ is a constant which will be chosen later, and $T^{*}>0$ ). We shall prove that

$$
c_{m} \leq \widetilde{c}
$$

where $\widetilde{c}$ is a constant independent of $m$ and $C$.

In fact, by Lemma 3.3 and (3.90) we have

$$
\left|\eta_{m}\right|_{\infty, \Omega^{T^{*}}} \leq\left.\left.\widetilde{\psi}_{6}\left(T^{* 1 / 2} C K_{0}\right)\right|_{\varrho_{0}}\right|_{\infty, \Omega} \leq K
$$

for sufficiently small $T^{*}=T^{*}\left(C K_{0}\right)$ (where $K$ is a constant independent of $\left.C K_{0}\right)$. Similarly

$$
\left|\gamma_{m}\right|_{\infty, \Omega^{T^{*}}} \leq \vartheta_{0}+T^{* 1 / 2} C K_{0} \leq K \quad \text { for sufficiently small } T^{*} .
$$

Hence there exists a bounded domain $V \subset \mathbb{R}_{+}^{2}$ such that $V_{m} \subset V$ for any $m$. Thus, we proved that $\|p\|_{C^{3}\left(\bar{V}_{m}\right)}+\left\|c_{v}\right\|_{C^{2}\left(\bar{V}_{m}\right)}$ is estimated by a constant independent of $m$ and $K_{0}$. In the same way we can prove that there exist bounded domains $G \subset \mathbb{R}^{9}$ and $Q \subset \mathbb{R}^{3} \times(0, T)$ such that $G_{m} \subset G$ and $Q_{m} \subset Q$ for any $m$. Thus we have proved (3.93).

Now inequalities (3.92)-(3.93) yield that for $t \leq T^{*}$ we have

$$
y_{m+1}(t) \leq \beta_{1}\left(t^{a} C K_{0}, b, \widetilde{c}, t\right) \exp \left[t \beta_{2}\left(t^{a} C K_{0}, C K_{0}, b, \widetilde{c}\right)\right] \leq C K_{0},
$$

if $C=C\left(K_{0}\right)$ is chosen so large that

$$
\beta_{1}(0, b, \widetilde{c}, 0)<C K_{0}
$$


and if $T^{*}$ is sufficiently small $\left(C\left(K_{0}\right)\right.$ in $(3.94)$ is a nondecreasing continuous function of $K_{0}$ ).

In this way we have shown that there exists a sufficiently large $C=$ $C\left(K_{0}\right)$ (satisfying (3.94)) such that $C\left(K_{0}\right)$ is a nondecreasing continuous function of $K_{0}$, and a sufficiently small $T^{*}>0$ such that for $t \leq T^{*}$ and for $m=0,1, \ldots$ we have

$$
y_{m}(t) \leq C K_{0} .
$$

(By (3.95) and (3.93) inequalities (3.48) and (3.60) with $w=u_{m}, \eta=\eta_{m}$ and $\beta=\gamma_{m}$ are satisfied with sufficiently small $\delta$ and sufficiently small $T^{*}$ independent of $m$.)

Now we prove the convergence of the sequence $\left\{u_{m}, \gamma_{m}, \eta_{m}\right\}$. Consider the following system of problems for the differences $U_{m+1}=u_{m+1}-u_{m}$, $\Gamma_{m+1}=\gamma_{m+1}-\gamma_{m}, H_{m}=\eta_{m}-\eta_{m-1}$ :

$$
\begin{aligned}
\eta_{m} \partial_{t} U_{m+1} & -\mu \nabla_{u_{m}}^{2} U_{m+1}-\nu \nabla_{u_{m}} \nabla_{u_{m}} \cdot U_{m+1} \\
= & -H_{m} \partial_{t} u_{m}-\mu\left(\nabla_{u_{m}}^{2}-\nabla_{u_{m-1}}^{2}\right) u_{m} \\
& -\nu\left(\nabla_{u_{m}} \nabla_{u_{m}} \cdot-\nabla_{u_{m-1}} \nabla_{u_{m-1}} \cdot\right) u_{m} \\
& +\nabla_{u_{m}} p\left(\eta_{m}, \gamma_{m}\right)-\nabla_{u_{m-1}} p\left(\eta_{m-1}, \gamma_{m-1}\right)+H_{m} g \\
\equiv & F_{1}+F_{2},
\end{aligned}
$$$$
\mathbb{D}_{u_{m}}\left(U_{m+1}\right) \cdot \bar{n}\left(u_{m}\right)
$$$$
=-\left[\mathbb{D}_{u_{m}}\left(u_{m}\right) \cdot \bar{n}\left(u_{m}\right)-\mathbb{D}_{u_{m-1}}\left(u_{m}\right) \cdot \bar{n}\left(u_{m-1}\right)\right]
$$$$
+\left[p\left(\eta_{m}, \gamma_{m}\right) \bar{n}\left(u_{m}\right)-p\left(\eta_{m-1}, \gamma_{m-1}\right) \bar{n}\left(u_{m-1}\right)\right]
$$$$
-p_{0}\left[\bar{n}\left(u_{m}\right)-\bar{n}\left(u_{m-1}\right)\right] \equiv G_{1}+G_{2} \text {, }
$$

$\left.U_{m+1}\right|_{t=0}=0$;

$$
\begin{aligned}
\eta_{m} c_{v} & \left(\eta_{m}, \gamma_{m}\right) \partial_{t} \Gamma_{m+1}-\kappa \nabla_{u_{m}}^{2} \Gamma_{m+1}=-H_{m} c_{v}\left(\eta_{m}, \gamma_{m}\right) \partial_{t} \gamma_{m} \\
& +\eta_{m-1} \partial_{t} \gamma_{m}\left[c_{v}\left(\eta_{m-1}, \gamma_{m-1}\right)-c_{v}\left(\eta_{m}, \gamma_{m}\right)\right] \\
& +\frac{\mu}{2} \sum_{i, j=1}^{3}\left[\left(\xi_{x_{i}} \cdot \nabla_{\xi} u_{m j}+\xi_{x_{j}} \cdot \nabla_{\xi} u_{m i}\right)^{2}\right. \\
& \left.-\left(\xi_{x_{i}} \cdot \nabla_{\xi} u_{m-1, j}+\xi_{x_{j}} \cdot \nabla_{\xi} u_{m-1, i}\right)^{2}\right] \\
& +(\nu-\mu)\left[\left(\nabla_{u_{m}} \cdot u_{m}\right)^{2}-\left(\nabla_{u_{m-1}} \cdot u_{m-1}\right)^{2}\right] \\
& -\gamma_{m} p_{\gamma}\left(\eta_{m}, \gamma_{m}\right) \nabla_{u_{m}} \cdot u_{m} \\
& +\gamma_{m-1} p_{\gamma}\left(\eta_{m-1}, \gamma_{m-1}\right) \nabla_{u_{m-1}} \cdot u_{m-1}+H_{m} k \equiv I_{1}+I_{2},
\end{aligned}
$$




$$
\begin{aligned}
& \bar{n}\left(u_{m}\right) \cdot \nabla_{u_{m}}\left(\Gamma_{m+1}\right) \\
& \quad=-\left[\bar{n}\left(u_{m}\right) \cdot \nabla_{u_{m}} \gamma_{m}-\bar{n}\left(u_{m-1}\right) \cdot \nabla_{u_{m-1}} \gamma_{m}\right] \equiv J, \\
& \left.\Gamma_{m}\right|_{t=0}=0 ; \\
& \partial_{t} H_{m}+H_{m} \operatorname{div}_{u_{m}} u_{m}=-\eta_{m-1}\left(\operatorname{div}_{u_{m}} u_{m}-\operatorname{div}_{u_{m-1}} u_{m-1}\right), \\
& \left.H_{m}\right|_{t=0}=0,
\end{aligned}
$$

where

$$
\begin{aligned}
& \operatorname{div}_{u_{i}} u_{i}=\nabla_{u_{i}} \cdot u_{i}, \\
& F_{2}=-H_{m} \partial_{t} u_{m}+H_{m} g+\nabla_{u_{m}} p\left(\eta_{m}, \gamma_{m}\right)-\nabla_{u_{m-1}} p\left(\eta_{m-1}, \gamma_{m-1}\right), \\
& G_{2}= p\left(\eta_{m}, \gamma_{m}\right) \bar{n}\left(u_{m}\right)-p\left(\eta_{m-1}, \gamma_{m-1}\right) \bar{n}\left(u_{m-1}\right), \\
& I_{2}=-H_{m} c_{v}\left(\eta_{m}, \gamma_{m}\right) \partial_{t} \gamma_{m}+\eta_{m-1} \partial_{t} \gamma_{m}\left[c_{v}\left(\eta_{m-1}, \gamma_{m-1}\right)-c_{v}\left(\eta_{m}, \gamma_{m}\right)\right] \\
&+H_{m} k+\gamma_{m-1} p_{\gamma}\left(\eta_{m-1}, \gamma_{m-1}\right) \nabla_{u_{m-1}} \cdot u_{m-1} \\
&-\gamma_{m} p_{\gamma}\left(\eta_{m}, \gamma_{m}\right) \nabla_{u_{m}} \cdot u_{m}
\end{aligned}
$$

and $F_{1}, G_{1}, I_{1}$ are determined by the remaining terms on the right-hand sides.

Applying Theorems 3.5, 3.6 and Lemma 3.3 to problems (3.96) and (3.97) respectively and using inequalities (3.93), (3.95) we obtain

$$
\begin{aligned}
\| U_{m+1} & \left\|_{4, \Omega^{t}}+\sup _{t}\left|U_{m+1}\right|_{3,0, \Omega}+\right\| \Gamma_{m+1} \|_{4, \Omega^{t}}+\sup _{t}\left|\Gamma_{m+1}\right|_{3,0, \Omega} \\
\leq & \widetilde{\phi}\left(t, K_{0}\right)\left[\left\|U_{m+1}\right\|_{2, \Omega^{t}}+\left\|F_{1}\right\|_{2, \Omega^{t}}+\left\|F_{2}\right\|_{2, \Omega^{t}}+\left\|I_{1}\right\|_{2, \Omega^{t}}\right. \\
& +\left\|I_{2}\right\|_{2, \Omega^{t}}+\left\|G_{1}\right\|_{3-1 / 2, S^{t}}+\left\|G_{2}\right\|_{3-1 / 2, S^{t}}+\mid D_{\xi, t}^{2} G_{1} \mathbf{|}_{1 / 4, S^{t}} \\
& \left.+\left|\mathbf{I} D_{\xi, t}^{2} G_{2} \mathbf{l}_{1 / 4, S^{t}}+\|J\|_{3-1 / 2, S^{t}}+\right| D_{\xi, t}^{2} J \mathbf{|}_{1 / 4, S^{t}}\right],
\end{aligned}
$$

where $\widetilde{\phi}$ is an nonnegative increasing continuous function of its arguments.

To estimate the right-hand sides of (3.99) we shall consider the following functions connected with the qualitative forms of $F_{i}, I_{i}, G_{i}(i=1,2)$ and $J$ :

$$
\begin{aligned}
F_{1}^{\prime}= & f_{1}\left(\mathbf{1}+\int_{0}^{t} D_{\xi}^{1} \widetilde{u}_{m} d \tau\right) \int_{0}^{t} D_{\xi}^{1} U_{m} d \tau D_{\xi}^{2} u_{m} \\
& +f_{2}\left(\mathbf{1}+\int_{0}^{t} D_{\xi}^{1} u_{m} d \tau, \mathbf{1}+\int_{0}^{t} D_{\xi}^{1} \widetilde{u}_{m} d \tau\right) \\
& \times \int_{0}^{t} D_{\xi}^{1} U_{m} d \tau \int_{0}^{t} D_{\xi}^{2} \widetilde{u}_{m} d \tau D_{\xi}^{1} u_{m}
\end{aligned}
$$




$$
\begin{aligned}
& +f_{3}\left(\mathbf{1}+\int_{0}^{t} D_{\xi}^{1} \widetilde{u}_{m} d \tau, \mathbf{1}+\int_{0}^{t} D_{\xi}^{1} u_{m-1} d \tau\right) \int_{0}^{t} D_{\xi}^{1} U_{m} d \tau \\
& \times \int_{0}^{t} D_{\xi}^{2} u_{m-1} d \tau D_{\xi}^{1} u_{m} \\
& F_{2}^{\prime}=-H_{m} \sum_{i=1}^{3} \partial_{t} u_{m i}+H_{m} \sum_{i=1}^{3} g_{i} \\
& +f_{4}\left(1+\int_{0}^{t} D_{\xi}^{1} \widetilde{u}_{m} d \tau\right) \\
& \times \int_{0}^{t} D_{\xi}^{1} U_{m} d \tau\left[p_{\eta}\left(\eta_{m}, \gamma_{m}\right) D_{\xi}^{1} \eta_{m}+p_{\gamma}\left(\eta_{m}, \gamma_{m}\right) D_{\xi}^{1} \gamma_{m}\right] \\
& +f_{5}\left(\mathbf{1}+\int_{0}^{t} D_{\xi}^{1} u_{m-1} d \tau\right)\left[p_{\eta \eta}\left(\widetilde{\eta}_{m}, \widetilde{\gamma}_{m}\right) D_{\xi}^{1} \widetilde{\eta}_{m} H_{m}\right. \\
& +p_{\eta \gamma}\left(\widetilde{\eta}_{m}, \widetilde{\gamma}_{m}\right) D_{\xi}^{1} \gamma_{m} H_{m}+p_{\gamma \eta}\left(\widetilde{\eta}_{m}, \widetilde{\gamma}_{m}\right) D_{\xi}^{1} \widetilde{\eta}_{m} \Gamma_{m} \\
& +p_{\gamma \gamma}\left(\widetilde{\eta}_{m}, \widetilde{\gamma}_{m}\right) D_{\xi}^{1} \widetilde{\gamma}_{m} \Gamma_{m} \\
& \left.+p_{\eta}\left(\widetilde{\eta}_{m}, \widetilde{\gamma}_{m}\right) D_{\xi}^{1} H_{m}+p_{\gamma}\left(\widetilde{\eta}_{m}, \widetilde{\gamma}_{m}\right) D_{\xi}^{1} \Gamma_{m}\right] ; \\
& I_{1}^{\prime}=f_{6}\left(\mathbf{1}+\int_{0}^{t} D_{\xi}^{1} u_{m} d \tau\right) D_{\xi}^{1} u_{m} D_{\xi}^{1} U_{m} \\
& +f_{7}\left(\mathbf{1}+\int_{0}^{t} D_{\xi}^{1} u_{m} d \tau, \mathbf{1}+\int_{0}^{t} D_{\xi}^{1} \widetilde{u}_{m} d \tau\right) \\
& \times D_{\xi}^{1} u_{m} \int_{0}^{t} D_{\xi}^{1} U_{m} d \tau D_{\xi}^{1} u_{m-1} \\
& +f_{8}\left(\mathbf{1}+\int_{0}^{t} D_{\xi}^{1} u_{m-1} d \tau\right) D_{\xi}^{1} U_{m} D_{\xi}^{1} u_{m-1} \\
& +f_{9}\left(\mathbf{1}+\int_{0}^{t} D_{\xi}^{1} \widetilde{u}_{m} d \tau, \mathbf{1}+\int_{0}^{t} D_{\xi}^{1} u_{m-1} d \tau\right) \\
& \times \int_{0}^{t} D_{\xi}^{1} U_{m} d \tau D_{\xi}^{1} u_{m-1} ; \\
& I_{2}^{\prime}=-H_{m} c_{v}\left(\eta_{m}, \gamma_{m}\right) \partial_{t} \gamma_{m}+H_{m} k \\
& +\eta_{m-1} \partial_{t} \gamma_{m}\left[c_{v \eta}\left(\widetilde{\eta}_{m}, \widetilde{\gamma}_{m}\right) H_{m}+c_{v \gamma}\left(\widetilde{\eta}_{m}, \widetilde{\gamma}_{m}\right) \Gamma_{m}\right]
\end{aligned}
$$




$$
\begin{aligned}
& +f_{10}\left(\mathbf{1}+\int_{0}^{t} D_{\xi}^{1} u_{m-1} d \tau\right) p_{\gamma}\left(\eta_{m-1}, \gamma_{m-1}\right) D_{\xi}^{1} u_{m-1} \Gamma_{m} \\
& +f_{11}\left(\mathbf{1}+\int_{0}^{t} D_{\xi}^{1} u_{m-1} d \tau\right) \\
& \times \gamma_{m}\left[p_{\gamma \eta}\left(\widetilde{\eta}_{m}, \widetilde{\gamma}_{m}\right) H_{m}+p_{\gamma \gamma}\left(\widetilde{\eta}_{m}, \widetilde{\gamma}_{m}\right) \Gamma_{m}\right] D_{\xi}^{1} u_{m-1} \\
& +f_{12}\left(\mathbf{1}+\int_{0}^{t} D_{\xi}^{1} u_{m-1} d \tau\right) \gamma_{m} p_{\gamma}\left(\eta_{m}, \gamma_{m}\right) D_{\xi}^{1} U_{m} \\
& +f_{13}\left(\mathbf{1}+\int_{0}^{t} D_{\xi}^{1} \widetilde{u}_{m} d \tau\right) \gamma_{m} p_{\gamma}\left(\eta_{m}, \gamma_{m}\right) \int_{0}^{t} D_{\xi}^{1} U_{m} d \tau D_{\xi}^{1} u_{m} \\
& G_{1}^{\prime}=f_{14}\left(\mathbf{1}+\int_{0}^{t} D_{\xi}^{1} u_{m-1} d \tau, \mathbf{1}+\int_{0}^{t} D_{\xi}^{1} \widetilde{u}_{m} d \tau\right) \\
& \times \int_{0}^{t} D_{\xi}^{1} U_{m} d \tau D_{\xi}^{1} u_{m} \\
& +f_{15}\left(\mathbf{1}+\int_{0}^{t} D_{\xi}^{1} u_{m} d \tau, \mathbf{1}+\int_{0}^{t} D_{\xi}^{1} \widetilde{u}_{m} d \tau\right) \\
& \times\left(\int_{0}^{t} D_{\xi}^{1} U_{m} d \tau\right)^{2}\left(D_{\xi}^{1} u_{m}\right)^{2} \\
& +f_{16}\left(1+\int_{0}^{t} D_{\xi}^{1} \widetilde{u}_{m} d \tau\right) \int_{0}^{t} D_{\xi}^{1} U_{m} d \tau \\
& G_{2}^{\prime}=f_{17}\left(\mathbf{1}+\int_{0}^{t} D_{\xi}^{1} \widetilde{u}_{m} d \tau\right) p\left(\eta_{m}, \gamma_{m}\right) \int_{0}^{t} D_{\xi}^{1} U_{m} d \tau \\
& +f_{18}\left(1+\int_{0}^{t} D_{\xi}^{1} u_{m} d \tau\right) \\
& \times\left[p_{\eta}\left(\widetilde{\eta}_{m}, \widetilde{\gamma}_{m}\right) H_{m}+p_{\gamma}\left(\widetilde{\eta}_{m}, \widetilde{\gamma}_{m}\right) \Gamma_{m}\right] \mathbf{1} ; \\
& J^{\prime}=f_{19}\left(\mathbf{1}+\int_{0}^{t} D_{\xi}^{1} u_{m} d \tau, \mathbf{1}+\int_{0}^{t} D_{\xi}^{1} u_{m-1} d \tau, \mathbf{1}+\int_{0}^{t} D_{\xi}^{1} \widetilde{u}_{m} d \tau\right) \\
& \times \int_{0}^{t} D_{\xi}^{1} U_{m} d \tau D_{\xi}^{1} \gamma_{m}
\end{aligned}
$$$$
\text { (3.100) }
$$$$
\text { [cont.] }
$$ 
Here $f_{i}(i=1, \ldots, 19)$ are certain smooth vector-valued functions of their arguments, $\widetilde{u}_{m}=\theta_{1} u_{m}+\left(1-\theta_{1}\right) u_{m-1}, \widetilde{\eta}_{m}=\theta_{2} \eta_{m}+\left(1-\theta_{2}\right) \eta_{m-1}, \widetilde{\gamma}_{m}=$ $\theta_{3} \gamma_{m}+\left(1-\theta_{3}\right) \gamma_{m-1}, 0<\theta_{i}<1(i=1,2,3)$ and the products of vectors in (3.100) are understood in the sense of (2.2).

In order to estimate the functions in (3.100) we use the Hölder and Minkowski inequalities, Lemma 2.1, Lemma 3.3, (3.91) and (3.92). Since the estimates of $\left\|F_{i}\right\|_{2, \Omega^{t}},\left\|G_{i}\right\|_{3-1 / 2, S^{t}}+\mid D_{\xi, t}^{2} G_{i} \mathbf{I}_{1 / 4, S^{t}},\left\|I_{i}\right\|_{2, \Omega^{t}}$ (where $i=1,2)$ and $\|J\|_{3-1 / 2, S^{t}}+\mid D_{\xi, t}^{2} J \mathbf{|}_{1 / 4, S^{t}}$ are the same as those of $\left\|F_{i}^{\prime}\right\|_{2, \Omega^{t}}$, $\left\|G_{i}^{\prime}\right\|_{3-1 / 2, S^{t}}+\mid D_{\xi, t}^{2} G_{i}^{\prime} \mathbf{|}_{1 / 4, S^{t}},\left\|I_{i}^{\prime}\right\|_{2, \Omega^{t}}$ (where $\left.i=1,2\right)$ and $\left\|J^{\prime}\right\|_{3-1 / 2, S^{t}}+$ $\mathbf{|} D_{\xi, t}^{2} J^{\prime} \mathbf{|}_{1 / 4, S^{t}}$ we get

$$
\begin{aligned}
& \left\|F_{1}\right\|_{2, \Omega^{t}} \leq c_{1}\left(t, K_{0}\right) t^{1 / 2} Y_{m}(t), \\
& \left\|F_{2}\right\|_{2, \Omega^{t}} \leq c_{2}\left(K_{0}\right) \sup _{\tau}\left|H_{m}\right|_{3,0, \Omega} \\
& +c_{3}\left(t, K_{0}\right) t^{1 / 2}\left(Y_{m}(t)+\sup _{\tau}\left|H_{m}\right|_{3,0, \Omega}\right), \\
& \left\|I_{1}\right\|_{2, \Omega^{t}} \leq c_{4}\left(t, K_{0}\right) t^{1 / 2} Y_{m}(t), \\
& \left\|I_{2}\right\|_{2, \Omega^{t}} \leq c_{5}\left(K_{0}\right)\left(\sup _{\tau}\left|H_{m}\right|_{\infty, \Omega}+\sup _{\tau}\left|\Gamma_{m}\right|_{\infty, \Omega}\right) \\
& +c_{6}\left(t, K_{0}\right) t^{1 / 2} Y_{m}(t) \\
& +c_{7}\left(K_{0}\right) t^{1 / 2}\left(\sup _{\tau}\left|H_{m}\right|_{3,0, \Omega}+\sup _{\tau}\left|\Gamma_{m}\right|_{3,0, \Omega}\right), \\
& \left\|G_{1}\right\|_{3-1 / 2, S^{t}}+\mathbf{|} D_{\xi, t}^{2} G_{1} \mathbf{I}_{1 / 4, S^{t}} \leq c_{8}\left(t, K_{0}\right) t^{1 / 4} Y_{m}(t), \\
& \left\|G_{2}\right\|_{3-1 / 2, S^{t}}+\mid D_{\xi, t}^{2} G_{2} \mathbf{I}_{1 / 4, S^{t}} \leq c_{9}\left(t, K_{0}\right) t^{1 / 4}\left(Y_{m}(t)+\sup _{\tau}\left|H_{m}\right|_{3,0, \Omega}\right), \\
& \|J\|_{3-1 / 2, S^{t}}+\mid D_{\xi, t}^{2} J \mathbf{|}_{1 / 4, S^{t}} \leq c_{10}\left(t, K_{0}\right) t^{1 / 4} Y_{m}(t),
\end{aligned}
$$

where $Y_{m}(t)=\left\|U_{m}\right\|_{4, \Omega^{T}}+\sup _{\tau}\left|U_{m}\right|_{3,0, \Omega}+\left\|\Gamma_{m}\right\|_{4, \Omega^{T}}+\sup _{\tau}\left|\Gamma_{m}\right|_{3,0, \Omega}$ and $c_{i}(i=1, \ldots, 10)$ are nonnegative increasing continuous functions of their arguments.

Now inequalities (3.99)-(3.101) yield

$$
\begin{aligned}
Y_{m+1}(t) & \leq \widetilde{\phi}\left(t, K_{0}\right)\left\|U_{m+1}\right\|_{2, \Omega^{t}}+\widetilde{\phi}_{1}\left(t, K_{0}\right) t^{1 / 4} Y_{m}(t) \\
& +\widetilde{\phi}_{2}\left(t, K_{0}\right)\left(\sup _{\tau}\left|H_{m}\right|_{3,0, \Omega}+\sup _{\tau}\left|H_{m}\right|_{\infty, \Omega}+\sup _{\tau}\left|\Gamma_{m}\right|_{\infty, \Omega}\right) .
\end{aligned}
$$

By Lemma 3.3,

$$
\sup _{\tau}\left|H_{m}\right|_{\infty, \Omega} \leq c \sup _{\tau}\left|H_{m}\right|_{3,0, \Omega}
$$


and since $\Gamma_{m}(\tau)=\int_{0}^{\tau} \partial_{s} \Gamma_{m}(s) d s$ we have

$$
\sup _{\tau}\left|\Gamma_{m}\right|_{\infty, \Omega} \leq c t^{1 / 2}\left\|\Gamma_{m}\right\|_{4, \Omega^{t}} .
$$

Next, consider problem (3.98). Integrating $(3.98)_{1}$ with respect to time we obtain

$$
\begin{aligned}
& H_{m}(\xi, t) \\
& =-\exp \left(-\int_{0}^{t} \operatorname{div}_{u_{m}} u_{m} d \tau\right) \\
& \quad \times \int_{0}^{t}\left[\eta_{m-1}\left(\operatorname{div}_{u_{m}} u_{m}-\operatorname{div}_{u_{m-1}} u_{m-1}\right) \exp \left(\int_{0}^{t^{\prime}} \operatorname{div}_{u_{m}} u_{m} d t^{\prime \prime}\right)\right] d t^{\prime} .
\end{aligned}
$$

Therefore, we have (see [15])

$$
\sup _{\tau}\left|H_{m}\right|_{3,0, \Omega} \leq \widetilde{\phi}_{3}\left(t, K_{0}\right) t^{1 / 2}\left\|U_{m}\right\|_{4, \Omega^{t}} .
$$

Taking into account (3.102)-(3.105) and the Gronwall inequality we get

$$
Y_{m+1}(t) \leq \widetilde{\phi}_{4}\left(t, K_{0}\right) t^{1 / 4} Y_{m}(t) .
$$

Hence by (3.106) and (3.105) for $t \leq T^{* *} \leq T^{*}$ (where $T^{* *}$ is sufficiently small) the sequence $\left\{\left(u_{m}, \gamma_{m}, \eta_{m}\right)\right\}$ converges to a limit $(u, \gamma, \eta) \in W_{2}^{4,2}\left(\Omega^{t}\right)$ $\times W_{2}^{4,2}\left(\Omega^{t}\right) \times C^{0}\left(0, t ; \Gamma_{0}^{3,3 / 2}(\Omega)\right)$ which is a solution of (3.1). Moreover, from Lemma 3.3 it follows that $\eta_{t} \in L_{2}\left(0, t ; W_{2}^{3}(\Omega)\right)$ and $\eta_{t t} \in L_{2}\left(0, t ; W_{2}^{1}(\Omega)\right)$.

Uniqueness of $(u, \gamma, \eta)$ can be proved in the standard way.

This completes the proof of the theorem.

4. Local existence in the case $\sigma \neq 0$. In the case $\sigma \neq 0$ problem (1.1) in the Lagrangian coordinates takes the form

$$
\begin{aligned}
& \eta u_{t}-\mu \nabla_{u}^{2} u-\nu \nabla_{u} \nabla_{u} \cdot u+\nabla_{u} p(\eta, \gamma)=\eta g \quad \text { in } \Omega^{T}, \\
& \eta_{t}+\eta \nabla_{u} \cdot u=0 \quad \text { in } \Omega^{T}, \\
& \eta c_{v}(\eta, \gamma) \gamma_{t}-\kappa \nabla_{u}^{2} \gamma=-\gamma p_{\gamma}(\eta, \gamma) \nabla_{u} \cdot u \\
& \text { (4.1) }+\frac{\mu}{2} \sum_{i, j=1}^{3}\left(\xi_{x_{i}} \cdot \nabla_{\xi} u_{j}+\xi_{x_{j}} \cdot \nabla_{\xi} u_{i}\right)^{2}+(\nu-\mu)\left(\nabla_{u} \cdot u\right)^{2}+\eta k \text { in } \Omega^{T}, \\
& \mathbb{T}_{u}(u, p) \cdot \bar{n}-\sigma \Delta_{S_{t}}(t) X_{u}(\xi, t)=-p_{0} \bar{n} \quad \text { on } S^{T}, \\
& \bar{n} \cdot \nabla_{u} \gamma=\widetilde{\gamma} \quad \text { on } S^{T}, \\
& \left.u\right|_{t=0}=v_{0},\left.\quad \eta\right|_{t=0}=\varrho_{0},\left.\quad \gamma\right|_{t=0}=\vartheta_{0} \quad \text { in } \Omega .
\end{aligned}
$$


The argument used to prove local existence of problem (4.1) is the same as in the case $\sigma=0$ (see Sect. 3). We consider the problem

$$
\begin{array}{ll}
\eta u_{t}-\mu \nabla_{w}^{2} u-\nu \nabla_{w} \nabla_{w} \cdot u=F & \text { in } \Omega^{T}, \\
\Pi_{0} \Pi \mathbb{D}_{w}(u) \cdot \bar{n}_{0}=G_{1} & \text { on } S^{T}, \\
\bar{n}_{0} \cdot \mathbb{D}_{w}(u) \cdot \bar{n}_{0}-\sigma \bar{n}_{0} \cdot \Delta_{S_{t}}(t) \int_{0}^{t} u(\tau) d \tau & \\
=G_{2}+\sigma \int_{0}^{t} H(\tau) d \tau & \text { on } S^{T}, \\
\left.u\right|_{t=0}=u_{0} & \text { in } \Omega,
\end{array}
$$

where $\Pi_{0}$ and $\Pi$ are the projections defined by $\Pi_{0} g=g-\left(g \cdot \bar{n}_{0}\right) \bar{n}_{0}$ and $\Pi g=g-(g \cdot \bar{n}) \bar{n}$, respectively.

The following theorem holds (see [15]).

THEOREM 4.1. Let

$S \in W_{2}^{4+1 / 2}, F \in W_{2}^{2,1}\left(\Omega^{T}\right), G_{i} \in W_{2}^{3-1 / 2,3 / 2-1 / 4}\left(S^{T}\right)$,

$\left|D_{\xi, t}^{2} G_{i}\right|_{1 / 4, S^{T}}<\infty(i=1,2)$,

$D_{\xi}^{\alpha} G_{1}(0)=0, D_{\xi}^{\alpha}\left(\bar{n}_{0} \cdot \mathbb{D}_{\xi}(u(0)) \cdot \bar{n}_{0}-G_{2}(0)\right)=0(|\alpha| \leq 1)$,

$u_{0} \in W_{2}^{3}(\Omega), H \in W_{2}^{2-1 / 2,1-1 / 4}\left(S^{T}\right)$,

$\eta \in L_{\infty}\left(0, T ; \Gamma_{0}^{2,1}(\Omega)\right) \cap C^{\alpha}(\Omega)(\alpha \in(0,1)), 1 / \eta \in L_{\infty}\left(\Omega^{T}\right)$,

$w \in W_{2}^{4,2}\left(\Omega^{T}\right) \cap C^{0}\left(0, T ; \Gamma_{0}^{3,3 / 2}(\Omega)\right), T<\infty$.

Assume that $T$ is so small that

$$
\begin{aligned}
& T^{a}\left(\|w\|_{4, \Omega^{T}}+|w(0)|_{3,0, \Omega}\right) \\
& \times \widetilde{\phi}\left(\int_{0}^{T}\left|w_{\xi}\right|_{\infty, \Omega} d t,|1 / \eta|_{\infty, \Omega^{T}},|\eta|_{\infty, \Omega^{T}},\|w\|_{4, \Omega^{T}},|w(0)|_{3,0, \Omega}, T\right) \leq \delta,
\end{aligned}
$$

where $\widetilde{\phi}$ is a nonnegative nondecreasing continuous function of its arguments, $a>0$ is a constant and $\delta>0$ is sufficiently small. Then there exists a unique solution $u \in W_{2}^{4,2}(\Omega) \cap C^{0}\left(0, T ; \Gamma_{0}^{3,3 / 2}(\Omega)\right)$ to problem (4.2) satisfying the estimate

$$
\begin{aligned}
\|u\|_{4, \Omega^{T}} \leq & \phi_{1}\left(|1 / \eta|_{\infty, \Omega^{T}},|\eta|_{\infty, \Omega^{T}}, T\right)\left[\|F\|_{2, \Omega^{T}}+\sum_{i=1}^{2}\left\|G_{i}\right\|_{3-1 / 2, S^{T}}\right. \\
& +\sum_{i=1}^{2}\left|D_{\xi, t}^{2} G_{i}\right|_{1 / 4, S^{T}}+\|H\|_{2-1 / 2, S^{T}} \\
& +\phi_{2}\left(|\eta|_{2,0, \infty, \Omega^{T}},\|\eta\|_{\left.C^{\alpha}\left(\Omega^{T}\right)\|u\|_{2, \Omega^{T}}\right]}\right. \\
& +\phi_{3}\left(|1 / \eta|_{\infty, \Omega^{T}}, T\right)|u(0)|_{3,0, \Omega}
\end{aligned}
$$


where $\phi_{i}(i=1,2,3)$ are nonnegative increasing continuous functions of their arguments.

Now consider the problems

$$
\begin{array}{cc}
\eta_{m} \partial_{t} u_{m+1}-\mu \nabla_{u_{m}}^{2} u_{m+1}-\nu \nabla_{u_{m}} \nabla_{u_{m}} \cdot u_{m+1} & \\
=-\nabla_{u_{m}} p\left(\eta_{m}, \gamma_{m}\right)+\eta_{m} g & \text { in } \Omega^{T}, \\
\Pi_{0} \Pi_{u_{m}} \mathbb{D}_{u_{m}}\left(u_{m+1}\right) \cdot \bar{n}\left(u_{m}\right)=0 & \text { on } S^{T},
\end{array}
$$

$$
\begin{array}{cl}
\bar{n}_{0} \cdot \mathbb{D}_{u_{m}}\left(u_{m+1}\right) \cdot \bar{n}\left(u_{m}\right)-\sigma \bar{n}_{0} \cdot \Delta_{m}(t) \int_{0}^{t} u_{m+1}(\tau) d \tau & \\
\quad=\bar{n}_{0} \cdot \bar{n}\left(u_{m}\right)\left[p\left(\eta_{m}, \gamma_{m}\right)-p_{0}\right]+\sigma \bar{n}_{0} \cdot \Delta_{m}(t) \xi & \text { on } S^{T}, \\
\left.u_{m+1}\right|_{t=0}=v_{0} & \text { in } \Omega ;
\end{array}
$$

$$
\begin{array}{rlrl}
\eta_{m} c_{v}\left(\eta_{m}, \gamma_{m}\right) \partial_{t} \gamma_{m+1}-\kappa \nabla_{u_{m}}^{2} \gamma_{m+1} & \\
= & -\gamma_{m} p_{\gamma}\left(\eta_{m}, \gamma_{m}\right) \nabla_{u_{m}} \cdot u_{m} & \\
& +\frac{\mu}{2} \sum_{i, j=1}^{3}\left(\xi_{x_{i}} \cdot \nabla_{\xi} u_{m j}+\xi_{x_{j}} \cdot \nabla_{\xi} u_{m i}\right)^{2} & \\
& +(\nu-\mu)\left(\nabla_{u_{m}} \cdot u_{m}\right)^{2}+\eta_{m} k & \\
\bar{n}\left(u_{m}\right) \cdot \nabla_{u_{m}} \gamma_{m+1}=\widetilde{\gamma} & \text { in } \Omega^{T}, \\
\left.\gamma_{m+1}\right|_{t=0}=\vartheta_{0} & \text { on } S^{T},
\end{array}
$$

$$
\begin{array}{ll}
\partial_{t} \eta_{m}+\eta_{m} \nabla_{u_{m}} \cdot u_{m}=0 & \text { in } \Omega^{T}, \\
\left.\eta_{m}\right|_{t=0}=\varrho_{0} & \text { in } \Omega .
\end{array}
$$

For the zero step functions $u_{0}, \gamma_{0}$ and $\eta_{0}$ we take the functions given by formulae (3.74)-(3.80).

Using Theorems 4.1 and 3.6 to problems (4.5) and (4.6), respectively and repeating the argument from the proof of Theorem 3.7 we obtain

THEOREM 4.2. Let

$S \in W_{2}^{4+1 / 2}, f \in C^{2,1}\left(\mathbb{R}^{3} \times[0, T]\right), r \in C^{2,1}\left(\mathbb{R}^{3} \times[0, T]\right)$,

$\widetilde{\vartheta} \in C^{2,1}\left(\mathbb{R}^{3} \times[0, T]\right), v_{0} \in W_{2}^{3}(\Omega), \vartheta_{0} \in W_{2}^{3}(\Omega)$,

$1 / \vartheta_{0} \in L_{\infty}(\Omega), \vartheta_{0}>0, \varrho_{0} \in W_{2}^{3}(\Omega), 1 / \varrho_{0} \in L_{\infty}(\Omega), \varrho_{0}>0$,

$c_{v} \in C^{2}\left(\mathbb{R}_{+}^{2}\right), c_{v}>0, p \in C^{3}\left(\mathbb{R}_{+}^{2}\right)$.

Moreover, assume that the following compatibility conditions are satisfied: (4.8) $D_{\xi}^{\alpha}\left[\mathbb{D}_{\xi}\left(v_{0}\right) \cdot \bar{n}_{0}-\left(p_{0}\left(\varrho_{0}, \vartheta_{0}\right)-p_{0}\right) \bar{n}_{0}-\sigma \Delta_{S_{t}}(0) \xi\right]=0, \quad|\alpha| \leq 1$, on $S$, 
and

$$
D_{\xi}^{\alpha}\left(\bar{n}_{0} \cdot \nabla_{\xi} \vartheta_{0}-\widetilde{\vartheta}(\xi, 0)\right)=0, \quad|\alpha| \leq 1, \text { on } S .
$$

Let $T^{*}$ be so small that $0<c_{1}\left(1-C K_{0} T^{*}\right)^{3} \leq \operatorname{det}\left\{x_{\xi}\right\} \leq c_{2}\left(1+C K_{0} T^{*}\right)^{3}$ (where $x(\xi, t)=\xi+\int_{0}^{t} u_{0}(\xi, \tau) d \tau$ for $t \leq T^{*}, u_{0}$ is given by $(3.74), K_{0}$ is given by (3.91) and the constant $C=C\left(K_{0}\right)$ which is a nondecreasing continuous function of $K_{0}$ is given by (3.94)). Then there exists $T^{* *}, 0<$ $T^{* *} \leq T^{*}$ such that for $T \leq T^{* *}$ there exists a unique solution $(u, \gamma, \eta) \in$ $W_{2}^{4,2}\left(\Omega^{T}\right) \times W_{2}^{4,2}\left(\Omega^{T}\right) \times C^{0}\left(0, t ; \Gamma_{0}^{3,3 / 2}(\Omega)\right)$ of problem $(4.1)$ and

$$
\begin{aligned}
& \|u\|_{4, \Omega^{T}}+\|\gamma\|_{4, \Omega^{T}} \leq C K_{0}, \\
& \sup _{t}\|\eta\|_{3, \Omega}+\sup _{t}\left\|\eta_{t}\right\|_{2, \Omega}+\left\|\eta_{t}\right\|_{L_{2}\left(0, T ; W_{2}^{3}(\Omega)\right)}+\left\|\eta_{t t}\right\|_{L_{2}\left(0, T ; W_{2}^{1}(\Omega)\right)} \\
& \leq \Phi_{1}\left(T, T^{a} K_{0}\right)\left\|\varrho_{0}\right\|_{3, \Omega}, \\
& |1 / \eta|_{\infty, \Omega^{T}}+|\eta|_{\infty, \Omega^{T}} \leq \Phi_{2}\left(T^{1 / 2} K_{0}\right)\left|1 / \varrho_{0}\right|_{\infty, \Omega}+\Phi_{3}\left(T^{1 / 2} K_{0}\right)\left|\varrho_{0}\right|_{\infty, \Omega},
\end{aligned}
$$

where $\Phi_{i}(i=1,2,3)$ are increasing continuous functions of their arguments, $a>0$.

\section{References}

[1] R. A. Adams, Sobolev Spaces, Academic Press, 1975.

[2] G. Allain, Small-time existence for the Navier-Stokes equations with a free surface, Appl. Math. Optim. 16 (1987), 37-50.

[3] O. V. Besov, V. P. Il'in and S. M. Nikol'skiŭ, Integral Representations of Functions and Imbedding Theorems, Nauka, Moscow, 1975 (in Russian).

[4] L. Landau and E. Lifschitz, Hydrodynamics, Nauka, Moscow, 1986 (in Russian).

[5] T. Nishida, Equations of fluid dynamics: free surface problems, Comm. Pure Appl. Math. 39 (1986), 221-238.

[6] P. Secchi and A. Valli, A free boundary problem for compressible viscous fluids, J. Reine Angew. Math. 341 (1983), 1-31.

[7] V. A. Solonnikov, A priori estimates for parabolic equations of second order, Trudy Mat. Inst. Steklov. 70 (1964), 133-212 (in Russian).

[8] - On boundary problems for linear parabolic systems of differential equations of general type, Trudy Mat. Inst. Steklov. 83 (1965) (in Russian); English transl.: Proc. Steklov Inst. Math. 83 (1967).

[9] - - On the solvability of the initial-boundary value problem for equations of motion of the viscous compressible fluid, Zap. Nauchn. Sem. LOMI 56 (1976), 128-142 (in Russian).

[10] - - On an unsteady motion of an isolated volume of a viscous incompressible fluid, Izv. Akad. Nauk SSSR Ser. Mat. 51 (5) (1987), 1065-1087 (in Russian).

[11] _-, On an initial-boundary value problem for the Stokes system which appears in free boundary problems, Trudy Mat. Inst. Steklov. 188 (1990), 150-188 (in Russian).

[12] V. Solonnikov and A. Tani, Free boundary problem for a viscous compressible flow with surface tension, in: Constantine Carathéodory: An International Tribute, T. M. Rassias (ed.), World Scientific, 1991, 1270-1303. 
[13] W. M. Zajączkowski, On local motion of a compressible barotropic viscous fluid bounded by a free surface, in: Partial Differential Equations, Banach Center Publ. 27, Inst. Math., Polish Acad. Sci., Warszawa, 1992, 511-553.

[14] -, On nonstationary motion of a compressible barotropic viscous fluid bounded by a free surface, Dissertationes Math. 324 (1993).

[15] - On nonstationary motion of a compressible barotropic viscous capillary fluid bounded by a free surface, to appear.

[16] - , Local existence of solutions for free boundary problems for viscous fluids, to appear.

INSTITUTE OF MATHEMATICS

TECHNICAL UNIVERSITY OF ŁÓDŹ

AL. POLITECHNIKI 11

90-924 ŁÓDŹ, POLAND
INSTITUTE OF MATHEMATICS POLISH ACADEMY OF SCIENCES

ŚNIADECKICH 8 00-950 WARSAW, POLAND

Reçu par la Rédaction le 20.4.1993 